\begin{tabular}{|c|l|}
\hline Title & Anti de Sitter horospherical flat timelike surfaces \\
\hline Author(s) & Chen, Liang; Izumiya, Shyuichi; Pei, DongHe; Saji, Kentaro \\
\hline Citation & $\begin{array}{l}\text { Science China: Mathematics, 57(9), 1841-1866 } \\
\text { https://doi.org/L0.1007/311425-014_4870-7 }\end{array}$ \\
\hline Issue Date & 201409-01 \\
\hline Doc URL & http://hdl.handle.net/2115/59744 \\
\hline Rights & The final publication is available at link.springer.com \\
\hline Type & article (author version) \\
\hline File Information & SCM_57_1841-.pdf \\
\hline
\end{tabular}

Instructions for use 


\title{
Anti de Sitter horospherical flat timelike surfaces
}

\author{
Liang Chen ${ }^{1}$, Shyuichi Izumiya ${ }^{2}$, Donghe $\mathrm{Pei}^{1}$ and Kentaro Saji ${ }^{3}$ \\ 1. School of Mathematics and Statistics, Northeast Normal University, \\ Changchun 130024, P. R. China \\ 2. Department of Mathematics, Faculty of Science, Hokkaido University, \\ Sapporo 060-0810, Japan \\ 3. Department of Mathematics, Faculty of Education, Gifu University, \\ Gifu 501-1193, Japan
}

\begin{abstract}
In this paper, we investigate a special timelike surfaces in Anti de Sitter 3-space. We call such a timelike surface an Anti de Sitter horospherical flat surface which belongs to a class of surfaces given by one parameter families of Anti de Sitter horocycle. We give a generic classification of singularities and study the geometric properties of such surfaces from the viewpoint of Legendrian singularity theory.
\end{abstract}

Keywords: Anti de Sitter 3-space; Timelike surface; AdS-horocycle; AdS-horocyclic surface; Singularities

2000 Mathematics Subject classification: 53A35; 57R45; 58K40

\section{Introduction}

This paper is written as one of the research projects on differential geometry of submanifolds in Anti de Sitter 3-space from the viewpoint of singularity theory $[2,3,4,5]$. It is well known that Minkowski space is a flat Lorentzian space form and de Sitter space is the Lorentzian space form with positive curvature. There are several articles for the study of submanifolds in these two Lorentzian space forms $[7,11,12,13,14,15,16,17,18]$. The Lorentzian space form with the negative curvature is called Anti de Sitter space which is a very important subject in Physics (the theory of general relativity, the string theory and the brane world scenario etc [23, 27, 28]). Mathematically, Anti de Sitter space is a Lorentzian analogue of the hyperbolic space. Since hyperbolic space has a lot of interesting properties, we expect that Anti de Sitter space has much more interesting properties. In [4] we have studied the general timelike surfaces in Anti de Sitter 3-space as an application of Legendrian singularity theory. For the study of the general timelike surfaces, the AdS-nullcone Gauss image $\mathbb{G}_{n}^{ \pm}$and the AdS-torus Gauss map $\widetilde{\mathbb{G}_{n}^{ \pm}}$play important roles [4]. We introduced the notions of the AdS-null Gauss-Kronecker curvature $K_{A d S n}^{ \pm}$and the AdS-torus Gauss-Kronecker curvature $\widetilde{K_{A d S t}^{ \pm}}$. These two Gauss-Kronecker curvature functions have the same zero sets. We remark that AdS-torus Gauss-Kronecker curvature is not a Lorentz invariant but it is an $S O(2) \times S O(2)$-invariant. It describes the contact of timelike surfaces 
with AdS-horospheres as a local property. However, we can show that the condition $\widetilde{K_{A d S t}^{ \pm}}=0$ is invariant under Lorentz motions (cf., Proposition 2.1).

In this paper we investigate a special kind of timelike surfaces in Anti de Sitter 3-space here. We call a timelike surface an Anti de Sitter horospherical flat sruface (briefly, AdS-horo-flat surfce) if $\widetilde{K_{A d S t}^{ \pm}} \equiv 0$ (cf., §3). So that the AdS-horo-flatness is invariant under the Lorentz motion. Moreover, the second author et al. investigated the horospherical flat surfaces in hyperbolic 3-space [19]. We study here the AdS-horo-flat surfaces by the similar method to the case of horospherical flat surfaces. However, the situation is quit different from the hyperbolic case. If we consider the AdS-horo-flat surfces without umbilical points, there exist two lines of curvature at each point and one of which corresponds to the vanishing AdS-torus principle curvature. We can assume, at least locally, that one of the lines of curvature is spacelike curve and another one is timelike curve. Therefore we should investigate the geometric properties of spacelike curves and timelike curves in Anti de Sitter 3-space as the first step of this research plan [5]. For the timelike curves, for example, we can construct some new invariants which can be used to describe the contact of timelike curves with AdS-horospheres. We also use these invariants to characterize a geometric object which is called an Anti de Sitter horocycle (briefly, AdS-horocycle) (cf., §2) and then we use the AdS-horocycle to obtain a surface named an Anti de Sitter horocyclic surface (briefly, AdS-horocyclic surface) which is givben as a one parameter family of AdS-horocycles. We can show that a AdS-horo-flat surfce is (at least locally) parametrized as an AdS-horocyclic surfaces (cf., Theorem 3.6). So that the main subject in this paper is the AdS-horo-flat AdS-horocyclic surface. In Euclidean space, surfaces with the vanishing Gauss curvature are developable surfaces which belong to a special class of ruled surfaces $[8,9]$. Therefore, AdS-horocyclic surfaces are one of the analogous notions with ruled surfaces. In this paper, we investigate geometric properties and singularities of AdS-horo-flat AdS-horocyclic surfaces.

We give a brief review of differential geometry of non-degenerate curves and timelike surfaces in Anti de Sitter 3-space in $\S 2$. A timelike surface with vanishing AdS-torus Gauss-Kronecker curvature has been defined and studied in $\S 3$. We construct a function by using the AdS-nullcone Gauss image. By using this function, we define a one-parameter family of AdS-horocycles. We show that a umbilically free AdS-horo-flat surfce is an AdS-horocyclic surface. Moreover, we prove that each AdS-horocycle is the line of curvature with the vanishing AdS-torus principle curvature, that is, the non-degenrate curve in Anti de Sitter 3-space. In $\S 4,5$, we give a classification of singularities of the AdS-horo-flat AdS-horocyclic surfaces and investigate the geometric meanings of these singularities. Moreover, we have shown a nice duality relation between $H_{1}^{3}$ and $\Lambda^{3}$ in [3]. By using this duality relation, we study two special classes of surfaces in $\Lambda^{3}$ in $\S 6$. We call these kinds of surfaces AdS-flat tangent nullcone circular surfaces and AdS-flat tangent nullcone hyperboloid respectively. We also give classifications of singularities of these two classes of surfaces. Finally, in $\S 7$, we give two examples of flat AdS-horocyclic surfaces to explain that our situation is very different from the ruled surfaces in Euclidean space.

We shall assume throughout the whole paper that all the maps and manifolds are $C^{\infty}$ unless the contrary is explicitly stated.

\section{Differential geometry in Anti de Sitter 3-space}

In this section we review the basic results of the local differential geometry of non-degenerate curves and timelike surfaces in Anti de Sitter 3-space which are introduced in the previous 
papers $[2,3,4,5]$. For details of Lorentzian geometry, see [25].

We adopt the Lorentzian model of the Anti de Sitter 3 -space. Let $\mathbb{R}^{4}=\left\{\left(x_{1}, \cdots, x_{4}\right) \mid x_{i} \in\right.$ $\mathbb{R}(i=1, \cdots, 4)\}$ be a 4 -dimensional vector space. For any vectors $\boldsymbol{x}=\left(x_{1}, \cdots, x_{4}\right)$ and $\boldsymbol{y}=\left(y_{1}, \cdots, y_{4}\right)$ in $\mathbb{R}^{4}$, the pseudo scalar product of $\boldsymbol{x}$ and $\boldsymbol{y}$ is defined to be $\langle\boldsymbol{x}, \boldsymbol{y}\rangle=$ $-x_{1} y_{1}-x_{2} y_{2}+x_{3} y_{3}+x_{4} y_{4}$. We call $\left(\mathbb{R}^{4},\langle\rangle,\right)$ a semi-Euclidean 4-space with index 2 and write $\mathbb{R}_{2}^{4}$ instead of $\left(\mathbb{R}^{4},\langle\rangle,\right)$. We say that a non-zero vector $\boldsymbol{x}$ in $\mathbb{R}_{2}^{4}$ is spacelike, null or timelike if $\langle\boldsymbol{x}, \boldsymbol{x}\rangle>0,\langle\boldsymbol{x}, \boldsymbol{x}\rangle=0$ or $\langle\boldsymbol{x}, \boldsymbol{x}\rangle<0$ respectively. The norm of the vector $\boldsymbol{x} \in \mathbb{R}_{2}^{4}$ is defined by $\|\boldsymbol{x}\|=\sqrt{|\langle\boldsymbol{x}, \boldsymbol{x}\rangle|}$. We denote the signature of a vector $\boldsymbol{x}$ by

$$
\operatorname{sign}(\boldsymbol{x})= \begin{cases}1 & \boldsymbol{x} \text { is spacelike } \\ 0 & \boldsymbol{x} \text { is null } \\ -1 & \boldsymbol{x} \text { is timelike }\end{cases}
$$

For a vector $\boldsymbol{n} \in \mathbb{R}_{2}^{4}$ and a real number $c$, we define the hyperplane with pseudo-normal $\boldsymbol{n}$ by $H P(\boldsymbol{n}, c)=\left\{\boldsymbol{x} \in \mathbb{R}_{2}^{4} \mid\langle\boldsymbol{x}, \boldsymbol{n}\rangle=c\right\}$. We call $H P(\boldsymbol{n}, c)$ a Lorentz hyperplane, a semi-Euclidean hyperplane with index 2 or a null hyperplane if $\boldsymbol{n}$ is timelike, spacelike or null respectively.

We now define Anti de Sitter 3-space (briefly, AdS 3-space) by $H_{1}^{3}=\left\{\boldsymbol{x} \in \mathbb{R}_{2}^{4} \mid\langle\boldsymbol{x}, \boldsymbol{x}\rangle=\right.$ $-1\}$, a unit pseudo 3-sphere with index 2 by $S_{2}^{3}=\left\{\boldsymbol{x} \in \mathbb{R}_{2}^{4} \mid\langle\boldsymbol{x}, \boldsymbol{x}\rangle=1\right\}$, and a closed nullcone with vertex $\boldsymbol{a}$ by $\Lambda_{a}=\left\{\boldsymbol{x} \in \mathbb{R}_{2}^{4} \mid\langle\boldsymbol{x}-\boldsymbol{a}, \boldsymbol{x}-\boldsymbol{a}\rangle=0\right\}$. In particular we call $\Lambda_{0}$ the nullcone at the origin. We also define the Lorentz torus by

$$
T_{1}^{2}=\left\{\boldsymbol{x}=\left(x_{1}, x_{2}, x_{3}, x_{4}\right) \in \Lambda_{0} \mid x_{1}^{2}+x_{2}^{2}=x_{3}^{2}+x_{4}^{2}=1\right\} .
$$

If a non-zero vector $\boldsymbol{x}=\left(x_{1}, x_{2}, x_{3}, x_{4}\right) \in \Lambda_{0}$, we have

$$
\widetilde{\boldsymbol{x}}= \pm \frac{1}{\sqrt{x_{1}^{2}+x_{2}^{2}}}\left(x_{1}, x_{2}, x_{3}, x_{4}\right)= \pm \frac{1}{\sqrt{x_{1}^{2}+x_{2}^{2}}} \boldsymbol{x} \in T_{1}^{2} .
$$

For any $\boldsymbol{x}_{1}, \boldsymbol{x}_{2}, \boldsymbol{x}_{3} \in \mathbb{R}_{2}^{4}$, we define a vector $\boldsymbol{x}_{1} \wedge \boldsymbol{x}_{2} \wedge \boldsymbol{x}_{3}$ by

$$
\boldsymbol{x}_{1} \wedge \boldsymbol{x}_{2} \wedge \boldsymbol{x}_{3}=\left|\begin{array}{cccc}
-\boldsymbol{e}_{1} & -\boldsymbol{e}_{2} & \boldsymbol{e}_{3} & \boldsymbol{e}_{4} \\
x_{1}^{1} & x_{1}^{2} & x_{1}^{3} & x_{1}^{4} \\
x_{2}^{1} & x_{2}^{2} & x_{2}^{3} & x_{2}^{4} \\
x_{3}^{1} & x_{3}^{2} & x_{3}^{3} & x_{3}^{4}
\end{array}\right|
$$

where $\left\{\boldsymbol{e}_{1}, \boldsymbol{e}_{2}, \boldsymbol{e}_{3}, \boldsymbol{e}_{4}\right\}$ is the canonical basis of $\mathbb{R}_{2}^{4}$ and $\boldsymbol{x}_{i}=\left(x_{i}^{1}, x_{i}^{2}, x_{i}^{3}, x_{i}^{4}\right)$. We can easily check that $\left\langle\boldsymbol{x}, \boldsymbol{x}_{1} \wedge \boldsymbol{x}_{2} \wedge \boldsymbol{x}_{3}\right\rangle=\operatorname{det}\left(\boldsymbol{x}, \boldsymbol{x}_{1}, \boldsymbol{x}_{2}, \boldsymbol{x}_{3}\right)$, so that $\boldsymbol{x}_{1} \wedge \boldsymbol{x}_{2} \wedge \boldsymbol{x}_{3}$ is pseudo-orthogonal to any $\boldsymbol{x}_{i}$ (for $i=1,2,3)$.

We now consider a surface given by the intersection of $H_{1}^{3}$ with the hyperplane $H P(\boldsymbol{n}, c)$. We call it a Anti de Sitter pseudohyperboloid with index 1 (briefly, AdS-pseudohyperboloid), a Anti de Sitter pseudosphere with index 1 (briefly, AdS-pseudosphere) or a Anti de Sitter horosphere (briefly, AdS-horosphere) if $\boldsymbol{n}$ is spacelike, timelike and $\|\boldsymbol{n}\|<|c|$ or null respectively. Especially, we denote a AdS-horosphere by $A H(\boldsymbol{n}, c)=H_{1}^{3} \cap H P(\boldsymbol{n}, c)$. If we consider a null vector $\boldsymbol{n}_{0}=-\boldsymbol{n} / c$, we have $A H(\boldsymbol{n}, c)=A H\left(\boldsymbol{n}_{0},-1\right)$. We call $\boldsymbol{n}_{0}$ the polar vector of $A H\left(\boldsymbol{n}_{0},-1\right)$.

We now review the extrinsic differential geometry on non-degenerate curves in $H_{1}^{3}$ (cf., [5]). Let $\gamma: I \longrightarrow H_{1}^{3}$ be a regular curve (i.e., an immersion). The regular curve $\gamma$ is said to be spacelike or timelike if $\dot{\gamma}$ is a spacelike or timelike vector at any $t \in I$, where $\dot{\gamma}=d \boldsymbol{\gamma} / d t$. We call such the a non-degenerate curve. Since $\gamma$ is a non-degenerate curve, it admits an arc length parametrization $s=s(t)$. Therefore, we can assume that $\gamma(s)$ is a unit speed curve. Now we have the unit tangent vector $\boldsymbol{t}(s)=\boldsymbol{\gamma}^{\prime}(s)$. Since $\langle\boldsymbol{\gamma}(s), \boldsymbol{\gamma}(s)\rangle \equiv-1$, we have $\langle\boldsymbol{\gamma}(s), \boldsymbol{t}(s)\rangle \equiv 0$. From a direct calculation we have $\left\langle\gamma(s), \boldsymbol{t}^{\prime}(s)\right\rangle=-\varepsilon$, where $\varepsilon=\operatorname{sign}(\boldsymbol{t}(s))$. We now denote $\mathbb{N}(s)=\boldsymbol{t}^{\prime}(s)-\varepsilon \boldsymbol{\gamma}(s)$ and $\mathbb{E}(s)=\boldsymbol{\gamma}(s) \wedge \boldsymbol{t}(s) \wedge \mathbb{N}(s)$. It is easy to check that $\mathbb{N}$ and $\mathbb{E}$ are 
pseudo-normal vectors along non-degenerate curve $\gamma$ in $H_{1}^{3}$. We also define the curvature by $k_{g}(s)=\left\|\boldsymbol{t}^{\prime}(s)-\varepsilon \boldsymbol{\gamma}(s)\right\|$. In the case when $k_{g}(s) \neq 0$, we can define unit vectors $\boldsymbol{n}(s)$ and $\boldsymbol{e}(s)$ by

$$
\boldsymbol{n}(s)=\frac{\boldsymbol{t}^{\prime}(s)-\varepsilon \boldsymbol{\gamma}(s)}{\left\|\boldsymbol{t}^{\prime}(s)-\varepsilon \boldsymbol{\gamma}(s)\right\|}=\frac{\mathbb{N}(s)}{\|\mathbb{N}(s)\|}, \quad \boldsymbol{e}(s)=\gamma(s) \wedge \boldsymbol{t}(s) \wedge \boldsymbol{n}(s) .
$$

Then we have a pseudo orthonormal frame $\{\boldsymbol{\gamma}(s), \boldsymbol{t}(s), \boldsymbol{n}(s), \boldsymbol{e}(s)\}$ of $\mathbb{R}_{2}^{4}$ along $\boldsymbol{\gamma}$. We assume $\operatorname{sign}(\boldsymbol{t}(s)) \operatorname{sign}(\boldsymbol{n}(s))=-1$ and give the following Frenet-Serret type formula by the standard arguments:

$$
\left\{\begin{array}{l}
\boldsymbol{\gamma}^{\prime}(s)=\boldsymbol{t}(s) \\
\boldsymbol{t}^{\prime}(s)=\varepsilon \boldsymbol{\gamma}(s)+k_{g}(s) \boldsymbol{n}(s) \\
\boldsymbol{n}^{\prime}(s)=k_{g}(s) \boldsymbol{t}(s)-\tau_{g}(s) \boldsymbol{e}(s) \\
\boldsymbol{e}^{\prime}(s)=-\varepsilon \tau_{g}(s) \boldsymbol{n}(s)
\end{array}\right.
$$

where $\tau_{g}(s)=\frac{1}{k_{g}^{2}(s)} \operatorname{det}\left(\boldsymbol{\gamma}(s), \gamma^{\prime}(s), \gamma^{\prime \prime}(s), \gamma^{\prime \prime \prime}(s)\right)$.

We remark that if $k_{g}(s)=0$ and $\mathbb{N}(s) \neq \mathbf{0}$, we have $\mathbb{N}(s)$ is a null vector. Since $\langle\mathbb{N}(s) \pm$ $\mathbb{E}(s), \mathbb{N}(s)\rangle=0$, it follows that $\mathbb{N}(s) \pm \mathbb{E}(s)$ is a null vector which is parallel to the vector $\mathbb{N}(s)$. Moreover we call $\gamma$ an Anti de Sitter horocycle (briefly, AdS-horocycle) if $k_{g}(s) \equiv 1$ and $\tau_{g}(x) \equiv 0$. Especially, we call $\boldsymbol{\gamma}$ a timelike Anti de Sitter horocycle (briefly, TAdS-horocycle) or spacelike Anti de Sitter horocycle (briefly, SAdS-horocycle) if $\boldsymbol{\gamma}$ is a timelike curve or spacelike curve respectively.

On the other hand, we outline the extrinsic differential geometry of timelike surfaces in Anti de Sitter 3-space which was developed in previous paper [4]. Let $\boldsymbol{X}: U \longrightarrow H_{1}^{3}$ be a regular surface (i.e., an embedding), where $U \subset \mathbb{R}^{2}$ is an open subset. We denote $M=\boldsymbol{X}(U)$ and identify $M$ with $U$ through the embedding $\boldsymbol{X}$. The embedding $\boldsymbol{X}$ is said to be timelike if the induced metric $\mathbf{I}$ of $M$ is Lorentzian. Throughout the remainder in this paper we assume that $M$ is a timelike surface in $H_{1}^{3}$. We define a vector $\boldsymbol{N}(u)$ by

$$
\boldsymbol{N}(u)=\frac{\boldsymbol{X}(u) \wedge \boldsymbol{X}_{u_{1}}(u) \wedge \boldsymbol{X}_{u_{2}}(u)}{\left\|\boldsymbol{X}(u) \wedge \boldsymbol{X}_{u_{1}}(u) \wedge \boldsymbol{X}_{u_{2}}(u)\right\|}
$$

By definition, we have $\langle\boldsymbol{N}(u), \boldsymbol{X}(u)\rangle \equiv\left\langle\boldsymbol{N}(u), \boldsymbol{X}_{u_{i}}(u)\right\rangle \equiv 0$ and $\left\langle\boldsymbol{X}(u), \boldsymbol{X}_{u_{i}}(u)\right\rangle \equiv 0$ (for $i=$ 1,2). This means that $\boldsymbol{X}(u), \boldsymbol{N}(u) \in N_{p} M$, where $u=\left(u_{1}, u_{2}\right) \in U$ and $p=\boldsymbol{X}(u) \in M$. Since the embedding is timelike and $\boldsymbol{X}(u) \in H_{1}^{3}, \boldsymbol{N}$ is spacelike. Therefore $\boldsymbol{N}(u) \in S_{2}^{3}$. It follows that $\boldsymbol{X}(u) \pm \boldsymbol{N}(u) \in \Lambda_{0} \cap N_{p} M$ and $\boldsymbol{X}(u) \pm \boldsymbol{N}(u) \in T_{1}^{2} \cap N_{p} M$. Thus we can define a map

$$
\mathbb{G}_{n}^{ \pm}: U \longrightarrow \Lambda_{0}
$$

by $\mathbb{G}_{n}^{ \pm}(u)=\boldsymbol{X}(u) \pm \boldsymbol{N}(u)$. This map is analogous to the hyperbolic Gauss indicatrix of hypersurfaces in $H_{+}^{n}(-1)$ which was defined in [10]. Here, we call it the Anti de Sitter nullcone Gauss image (briefly, AdS-nullcone Gauss image) of $\boldsymbol{X}$ (or $M$ ). We also define a map

$$
\widetilde{\mathbb{G}_{n}^{ \pm}}: U \longrightarrow T_{1}^{2}
$$

by $\widetilde{\mathbb{G}_{n}^{ \pm}}(u)=\boldsymbol{X}\left(\widetilde{\bar{N} \pm \boldsymbol{N}}(u)=\frac{1}{\xi(u)} \mathbb{G}_{n}^{ \pm}(u)\right.$, where $\xi(u)= \pm \sqrt{\left(x_{1}(u) \pm n_{1}(u)\right)^{2}+\left(x_{2}(u) \pm n_{2}(u)\right)^{2}}$, $\boldsymbol{X}(u)=\left(x_{1}(u), x_{2}(u), x_{3}(u), x_{4}(u)\right)$ and $\boldsymbol{N}(u)=\left(n_{1}(u), n_{2}(u), n_{3}(u), n_{4}(u)\right)$. We call it the Anti de Sitter torus Gauss map (or, AdS-torus Gauss map) of $\boldsymbol{X}$.

We remark that the map $\mathbb{G}_{n}^{ \pm}(u)$ was used by S. Lee [22] to study the timelike sufaces of constant mean curvature \pm 1 in Anti de Sitter 3 -space. He called $\mathbb{G}_{n}^{ \pm}(u)$ the hyperbolic Gauss map. By a direct calculation we know that $\mathbb{G}_{n}^{ \pm}$is constant if and only if $\widetilde{\mathbb{G}_{n}^{ \pm}}$is constant. We 
have shown that the timelike surface $\boldsymbol{X}=M$ is a part of AdS-horosphere if and only if $\mathbb{G}_{n}^{ \pm}$is constant.

It is easy to show that $\boldsymbol{N}_{u_{i}}(i=1,2)$ are tangent vectors of $M$. Therefore we have a linear transformation

$$
S_{p}^{ \pm}=-d \mathbb{G}_{n}^{ \pm}(u)=-(d \boldsymbol{X}(u) \pm d \boldsymbol{N}(u)): T_{p} M \longrightarrow T_{p} M
$$

which is called the Anti de Sitter null shape operator (briefly, AdS-null shape operator) of $M=$ $\boldsymbol{X}(U)$ at $p=\boldsymbol{X}(u)$. Under the identification of $U$ and $M$, the derivation $d \boldsymbol{X}(u)$ can be identified with the identity mapping $\operatorname{id}_{T_{p} M}$, this means that $S_{p}^{ \pm}=-d \mathbb{G}_{n}^{ \pm}(u)=-\left(\operatorname{id}_{T_{p} M} \pm d \boldsymbol{N}(u)\right)$. We have another linear mapping $d \widetilde{\mathbb{G}_{n}^{ \pm}}(u): T_{p} M \longrightarrow T_{p} \mathbb{R}_{2}^{4}=T_{p} M \oplus N_{p} M$. If we consider the orthogonal projection $\pi^{T}: T_{p} M \oplus N_{p} M \longrightarrow T_{p} M$, then we have

$$
\widetilde{S_{p}^{ \pm}}=-\left(d \widetilde{\mathbb{G}_{n}^{ \pm}}(u)\right)^{T}=-\pi^{T} \circ d \widetilde{\mathbb{G}_{n}^{ \pm}}(u): T_{p} M \longrightarrow T_{p} M
$$

and call it the Anti de Sitter torus shape operator (briefly, AdS-torus shape operator) of $M=$ $\boldsymbol{X}(U)$ at $p=\boldsymbol{X}(u)$. We remark that $S_{p}^{ \pm}$(resp., $\widetilde{S_{p}^{ \pm}}$) does not always have real eigenvalues. If the eigenvalues are real numbers, we denote it by $k_{i}^{ \pm}$(resp., $\widetilde{k_{i}^{ \pm}}$) (for $i=1,2$ ) and call it AdS-null principle curvature (resp., AdS-torus principle curvature).

We define $K_{A d S n}^{ \pm}(u)=\operatorname{det} S_{p}^{ \pm}=k_{1}^{ \pm} \cdot k_{2}^{ \pm}$and $\widetilde{K_{A d S t}^{ \pm}}(u)=\operatorname{det} \widetilde{S_{p}^{ \pm}}=\widetilde{k_{1}^{ \pm}} \cdot \widetilde{k_{2}^{ \pm}}$. We respectively call $K_{A d S n}^{ \pm}(u)$ the Anti de Sitter null Gauss-Kronecker curvature (briefly, AdS-null G-K curvature) and $\widetilde{K_{A d S t}^{ \pm}}(u)$ the Anti de Sitter torus Gauss-Kronecker curvature (briefly, AdS-torus G-K curvature) of $M=\boldsymbol{X}(U)$ at $p=\boldsymbol{X}(u)$. By a straightforward calculation we have the relation $S_{p}^{ \pm}=\xi(u) \widetilde{S_{p}^{ \pm}}$, so that we have $k_{i}^{ \pm}(p)=\xi(u) \widetilde{k_{i}^{ \pm}}(p)$ and $K_{A d S n}^{ \pm}(u)=\xi^{2}(u) \widetilde{K_{A d S t}^{ \pm}}(u)$. Then we have the following relations:

$$
\left\{\begin{array}{l}
k_{i}^{ \pm}(p)=0 \Longleftrightarrow \widetilde{k_{i}^{ \pm}}(u)=0 \\
K_{A d S n}^{ \pm}(u)=0 \Longleftrightarrow \widetilde{K_{A d S t}^{ \pm}}(u)=0 .
\end{array}\right.
$$

We say that a point $p=\boldsymbol{X}(u)$ is a (positive or negative) Anti de Sitter horospherical parabolic point (briefly, $A d S h^{ \pm}$-parabolic point) of $M=\boldsymbol{X}(U)$ if $\widehat{K_{A d S t}^{ \pm}}(u)=0\left(\right.$ or $\left.K_{A d S n}^{ \pm}(u)=0\right)$. We remark that the AdS-torus G-K curvature is not a Lorentz invariant but it is an $S O(2) \times S O(2)$ invariant. Moreover, we have the following:

Proposition 2.1 For a point $p=\boldsymbol{X}(u), \widetilde{k_{i}^{ \pm}}(p)$ is invariant under Lorentz motions if and only if $\widetilde{k_{i}^{ \pm}}(p)=0$.

Proof. We have the relation $\widetilde{k_{i}^{ \pm}}(p)=\frac{1}{\xi(u)} k_{i}^{ \pm}(p)$ at any point $p \in M$. Since $k_{i}^{ \pm}(p)$ is a Lorentz invariant, it is zero if and only if $\widetilde{k_{i}^{ \pm}}(p)$ is a Lorentz invariant.

We say that a point $u \in U$ or $p=\boldsymbol{X}(u)$ is an umbilic point if $S_{p}^{ \pm}=k^{ \pm}(p) i d_{T_{p} M}$. We also say that $M=\boldsymbol{X}(U)$ is totally umbilic if all points on $M$ are umbilic. We have the following classification theorem of totally umbilical timelike surfaces (cf. [4]):

Proposition 2.2 Suppose that $M=\boldsymbol{X}(U)$ is totally umbilic. Then $k^{ \pm}(p)$ is constant $k^{ \pm}$. Under this condition, we have the following classification.

(1) Suppose $k^{ \pm} \neq 0$.

(a) If $0<\left|k^{ \pm}+1\right|<1$, then $M$ is a part of an AdS-pseudohyperboloid;

(b) If $\left|k^{ \pm}+1\right|>1$, then $M$ is a part of an AdS-pseudosphere; 
(c) If $k^{ \pm}=-1$, then $M$ is a part of an AdS-small pseudohyperboloid.

(2) Suppose $k^{ \pm}=0$ then $M$ is a part of an AdS-horosphere.

We also call a point $p \in M$ the Anti de Sitter horospherical point (briefly, AdS-horospherical point $)$ if $k_{i}^{ \pm}(p)=0(i=1,2)$. By this proposition we have shown that a AdS-horosphere is a totally umbilical timelike surface with $k^{ \pm}=0$.

Corollary 2.3 If $M$ is totally umbilical and $\widetilde{k_{i}^{ \pm}}(p)=(1 / \xi(u)) k_{i}^{ \pm}(p)$ is a Lorentz invariant, then $M$ is a part of a AdS-horosphere.

We now introduce the Lorentz metric $d s^{2}=\sum_{i, j=1}^{2} g_{i j} d u_{i} d u_{j}$ on $M=\boldsymbol{X}(U)$, where $g_{i j}(u)=\left\langle\boldsymbol{X}_{u_{i}}(u), \boldsymbol{X}_{u_{j}}(u)\right\rangle$ for any $u \in U$. We also define the Anti de Sitter null second fundamental invariant by $h_{i j}^{ \pm}(u)=\left\langle-\left(\mathbb{G}_{n}^{ \pm}\right)_{u_{i}}(u), \boldsymbol{X}_{u_{j}}(u)\right\rangle$, the Anti de Sitter torus second fundamental invariant by $\widetilde{h_{i j}^{ \pm}}(u)=\left\langle-\left(\widetilde{\mathbb{G}_{n}^{ \pm}}\right)_{u_{i}}(u), \boldsymbol{X}_{u_{j}}(u)\right\rangle=\frac{1}{\xi(u)} h_{i j}^{ \pm}(u)$ for any $u \in U$. We can also show the following Weingarten formulas in [4].

Proposition 2.4 With the above notations the following hold

(1) AdS-null Weingarten formula

$$
\mathbb{G}_{n u_{i}}^{ \pm}=-\sum_{j=1}^{2}\left(h^{ \pm}\right)_{i}^{j} \boldsymbol{X}_{u_{j}}, \text { where }\left(\left(h^{ \pm}\right)_{i}^{j}\right)=\left(h_{i k}^{ \pm}\right)\left(g^{k j}\right) \text { and }\left(g^{k j}\right)=\left(g_{k j}\right)^{-1} .
$$

(2) AdS-torus Weingarten formula

$$
\left.\left(\widetilde{\mathbb{G}_{n}^{ \pm} u_{i}}\right)_{t}=-\sum_{j=1}^{2}\left(\widetilde{h^{ \pm}}\right)_{i}^{j} \boldsymbol{X}_{u_{j}} \text {, where }\left(\widetilde{h^{ \pm}}\right)_{i}^{j}\right)=\left(\widetilde{h_{i k}^{ \pm}}\right)\left(g^{k j}\right) \text { and }\left(g^{k j}\right)=\left(g_{k j}\right)^{-1} \text {. }
$$

As a corollary of the above proposition, we have the following expression of the AdS-null G-K curvature and AdS-torus G-K curvature .

Corollary 2.5 With the same notations as in the above Proposition, we have:

$$
K_{A d S n}^{ \pm}=\frac{\operatorname{det}\left(h_{i j}^{ \pm}\right)}{\operatorname{det}\left(g_{i j}\right)}=\xi^{2} \frac{\operatorname{det}\left(\widetilde{h_{i j}^{ \pm}}\right)}{\operatorname{det}\left(g_{i j}\right)}=\xi^{2} \widetilde{K_{A d S t}^{ \pm}} .
$$

\section{The AdS-horo-flat surfces}

In this section we consider timelike surfaces with vanishing AdS-torus (AdS-null) G-K curvature. At each point of the timelike surface, we have two different directed AdS-nullcone Gauss images $\mathbb{G}_{n}^{ \pm}$. Without loss of the generality, we only consider $\mathbb{G}_{n}^{+}=\boldsymbol{X}+\boldsymbol{N}=\mathbb{G}_{n}$. The other corresponding notations are also written in the similar way. We say that a timelike surface $M=\boldsymbol{X}(U)$ is an andti de Sitter horospherical flat surface (briefly, AdS-horo-flat surfce) if $K_{A d S n}^{ \pm}(p)=\widehat{K_{A d S t}^{ \pm}}(p)=0$ at any point $p \in M$. By this definition, the AdS-horosphere is one of the typical totally umbilical AdS-horo-flat surfce. Now we consider an umbilically free timelike surface $\boldsymbol{X}: U \longrightarrow H_{1}^{3}$, where $U \subset \mathbb{R}^{2}$ is a neighborhood around the origin. In this case, we have two kinds of lines of curvature, one of them is timelike curve, another one is spacelike curve. We assume that the $u$-curves correspond to the vanishing AdS-null principle curvature for the coordinate system $(u, v) \in U$. Firstly, we consider the case when the $u$-curves are timelike. By the AdS-null Weingarten formula, we have

$$
\mathbb{G}_{n u}(u, v)=0, \mathbb{G}_{n v}(u, v)=-k(u, v) \boldsymbol{X}_{v}(u, v),
$$


where $k(u, v) \neq 0$. It follows that $\mathbb{G}_{n}(0, v)=\mathbb{G}_{n}(u, v)$. We now define a function

$$
F: H_{1}^{3} \times(-\varepsilon, \varepsilon) \longrightarrow \mathbb{R}
$$

by $F(\boldsymbol{x}, v)=\left\langle\mathbb{G}_{n}(0, v), \boldsymbol{x}\right\rangle+1$ for sufficiently small $\varepsilon>0$. For any $v \in(-\varepsilon, \varepsilon)$, we have a AdS-horosphere $A H\left(\mathbb{G}_{n}\left(0, v_{0}\right),-1\right)$, so that $F=0$ define a one-parameter family of AdShorospheres. By almost the same arguments as those of [19], we have the following proposition.

Proposition 3.1 The timelike surface $M=\boldsymbol{X}(U)$ is the envelope of the family of AdShorospheres defined by $F=0$.

Moreover, we consider a timelike surface $\overline{\boldsymbol{X}}: I \times J \longrightarrow H_{1}^{3}$ defined by

$$
\overline{\boldsymbol{X}}(s, v)=\boldsymbol{X}(0, v)+s \frac{\boldsymbol{X}_{u}(0, v)}{\left\|\boldsymbol{X}_{u}(0, v)\right\|}-\frac{s^{2}}{2} \mathbb{G}_{n}(0, v)
$$

where $I, J \subset \mathbb{R}$ are open intervals. We have the following proposition.

Proposition 3.2 The timelike surface $\bar{M}=\overline{\boldsymbol{X}}(I \times J)$ is the envelope of the family of AdShorospheres defined by $F=0$.

The proof is almost the same as that of Proposition 4.2 in [19], so that we omit it. By the above two propositions, we have shown that a AdS-horo-flat surfce can be reparametrized (at least locally) by

$$
\overline{\boldsymbol{X}}(s, v)=\boldsymbol{X}(0, v)+s \frac{\boldsymbol{X}_{u}(0, v)}{\left\|\boldsymbol{X}_{u}(0, v)\right\|}-\frac{s^{2}}{2} \mathbb{G}_{n}(0, v) .
$$

We now give the expression of the above parametrization. For any fixed $v=v_{0}$, we assume that

$$
\boldsymbol{a}_{1}=\boldsymbol{X}\left(0, v_{0}\right), \boldsymbol{a}_{2}=\frac{\boldsymbol{X}_{u}\left(0, v_{0}\right)}{\left\|\boldsymbol{X}_{u}\left(0, v_{0}\right)\right\|} \in H_{1}^{3}, \boldsymbol{a}_{3}=\boldsymbol{N}\left(0, v_{0}\right) \in S_{2}^{3} .
$$

Then we can define a curve by

$$
\gamma(s)=\boldsymbol{a}_{1}+s \boldsymbol{a}_{2}-\frac{s^{2}}{2}\left(\boldsymbol{a}_{1}+\boldsymbol{a}_{3}\right)
$$

Since $\boldsymbol{\gamma}^{\prime}(s)=\boldsymbol{a}_{2}-s\left(\boldsymbol{a}_{1}+\boldsymbol{a}_{3}\right)$, we have $\left\langle\boldsymbol{\gamma}^{\prime}(s), \boldsymbol{\gamma}^{\prime}(s)\right\rangle=-1$. This means that the curve $\boldsymbol{\gamma}$ is a unit speed timelike curve. Therefore $\boldsymbol{t}(s)=\boldsymbol{a}_{2}-s\left(\boldsymbol{a}_{1}+\boldsymbol{a}_{3}\right)$. Moreover, $\boldsymbol{t}^{\prime}(s)=-\left(\boldsymbol{a}_{1}+\boldsymbol{a}_{3}\right)$, so that $\left\langle\boldsymbol{t}^{\prime}(s), \boldsymbol{t}^{\prime}(s)\right\rangle=0 \neq-1$. By a straight forward calculation, we have $\left\langle\boldsymbol{t}^{\prime}(s)+\boldsymbol{\gamma}(s), \boldsymbol{t}^{\prime}(s)+\boldsymbol{\gamma}(s)\right\rangle=1$ which is equivalent to the condition that $k_{g}(s)=1$. Moreover, since $\boldsymbol{\gamma}^{\prime \prime \prime}=0$, we have $\tau_{g}(s)=0$. Therefore, $\boldsymbol{\gamma}$ is a TAdS-horocycle. Since $\boldsymbol{\gamma}(0)=\boldsymbol{a}_{1}, \boldsymbol{\gamma}^{\prime}(0)=\boldsymbol{a}_{2}, \boldsymbol{\gamma}^{\prime \prime}(0)=-\left(\boldsymbol{a}_{1}+\boldsymbol{a}_{3}\right)$, we have the unique solution of the natural equation $k_{g}(s)=1, \tau_{g}(s)=0$ under the above initial data. Therefore we have the following proposition.

Proposition 3.3 For any $\boldsymbol{a}_{1}, \boldsymbol{a}_{2} \in H_{1}^{3}$ and $\boldsymbol{a}_{3} \in S_{2}^{3}$ such that $\left\langle\boldsymbol{a}_{i}, \boldsymbol{a}_{j}\right\rangle=0$, the unique TAdShorocycle with the initial conditions $\gamma(0)=\boldsymbol{a}_{1}, \gamma^{\prime}(0)=\boldsymbol{a}_{2}, \gamma^{\prime \prime}(0)=-\left(\boldsymbol{a}_{1}+\boldsymbol{a}_{3}\right)$ is given by $\gamma(s)=\boldsymbol{a}_{1}+s \boldsymbol{a}_{2}-\frac{s^{2}}{2}\left(\boldsymbol{a}_{1}+\boldsymbol{a}_{3}\right)$

On the other hand, we consider the case when the $u$-curves are spacelike. We define a timelike surface $\boldsymbol{X}^{\sharp}: I \times J \longrightarrow H_{1}^{3}$ by

$$
\boldsymbol{X}^{\sharp}(s, v)=\boldsymbol{X}(0, v)+s \frac{\boldsymbol{X}_{u}(0, v)}{\left\|\boldsymbol{X}_{u}(0, v)\right\|}+\frac{s^{2}}{2} \mathbb{G}_{n}(0, v),
$$


where $I, J \subset \mathbb{R}$ are open intervals. By the similar arguments as those in the proof of Proposition 3.2 , we have the following proposition.

Proposition 3.4 The timelike surface $M^{\sharp}=\boldsymbol{X}^{\sharp}(I \times J)$ is the envelope of the family of AdS-horospheres defined by $F=0$.

We have shown that a AdS-horo-flat surfce can be reparametrized (at least locally) by

$$
\boldsymbol{X}^{\sharp}(s, v)=\boldsymbol{X}(0, v)+s \frac{\boldsymbol{X}_{u}(0, v)}{\left\|\boldsymbol{X}_{u}(0, v)\right\|}+\frac{s^{2}}{2} \mathbb{G}_{n}(0, v) .
$$

We now consider the meaning of the above parametrization. For any fixed $v=v_{0}$, we assume that

$$
\boldsymbol{a}_{1}=\boldsymbol{X}\left(0, v_{0}\right) \in H_{1}^{3}, \boldsymbol{a}_{3}=\boldsymbol{N}\left(0, v_{0}\right), \boldsymbol{a}_{4}=\frac{\boldsymbol{X}_{u}\left(0, v_{0}\right)}{\left\|\boldsymbol{X}_{u}\left(0, v_{0}\right)\right\|} \in S_{2}^{3} .
$$

Then we can define a curve by

$$
\gamma(s)=\boldsymbol{a}_{1}+s \boldsymbol{a}_{4}+\frac{s^{2}}{2}\left(\boldsymbol{a}_{1}+\boldsymbol{a}_{3}\right)
$$

Since $\boldsymbol{\gamma}^{\prime}(s)=\boldsymbol{a}_{4}+s\left(\boldsymbol{a}_{1}+\boldsymbol{a}_{3}\right)$, we have $\left\langle\boldsymbol{\gamma}^{\prime}(s), \boldsymbol{\gamma}^{\prime}(s)\right\rangle=1$. This means that the curve $\boldsymbol{\gamma}$ is a unit speed spacelike curve. Therefore $\boldsymbol{t}(s)=\boldsymbol{a}_{4}+s\left(\boldsymbol{a}_{1}+\boldsymbol{a}_{3}\right)$. Moreover, $\boldsymbol{t}^{\prime}(s)=\boldsymbol{a}_{1}+\boldsymbol{a}_{3}$, so that $\left\langle\boldsymbol{t}^{\prime}(s), \boldsymbol{t}^{\prime}(s)\right\rangle=0 \neq-1$. By directly calculation, we have $\left\langle\boldsymbol{t}^{\prime}(s)-\boldsymbol{\gamma}(s), \boldsymbol{t}^{\prime}(s)-\boldsymbol{\gamma}(s)\right\rangle=1$ which is equivalent to the condition that $k_{g}(s)=1$. Moreover, since $\boldsymbol{\gamma}^{\prime \prime \prime}=0$, we have $\tau_{g}(s)=0$. Therefore, $\boldsymbol{\gamma}$ is a SAdS-horocycle. Since $\gamma(0)=\boldsymbol{a}_{1}, \boldsymbol{\gamma}^{\prime}(0)=\boldsymbol{a}_{4}, \boldsymbol{\gamma}^{\prime \prime}(0)=\boldsymbol{a}_{1}+\boldsymbol{a}_{3}$, we have the unique solution of the natural equation $k_{g}(s)=1, \tau_{g}(s)=0$ under the above initial data. Therefore we have the following proposition similar as Proposition 3.3.

Proposition 3.5 For any $\boldsymbol{a}_{1} \in H_{1}^{3}$ and $\boldsymbol{a}_{3}, \boldsymbol{a}_{4} \in S_{2}^{3}$ such that $\left\langle\boldsymbol{a}_{i}, \boldsymbol{a}_{j}\right\rangle=0$, the unique SAdS-horocycle with the initial conditions $\boldsymbol{\gamma}(0)=\boldsymbol{a}_{1}, \boldsymbol{\gamma}^{\prime}(0)=\boldsymbol{a}_{4}, \boldsymbol{\gamma}^{\prime \prime}(0)=\boldsymbol{a}_{1}+\boldsymbol{a}_{3}$ is given by $\gamma(s)=\boldsymbol{a}_{1}+s \boldsymbol{a}_{4}+\frac{s^{2}}{2}\left(\boldsymbol{a}_{1}+\boldsymbol{a}_{3}\right)$

By the above proposition and Proposition 3.3, we have shown that the AdS-horo-flat surfce is given by the one-parameter family of AdS-horocycle. We call a timelike surface an $A d S$ horocyclic surface if it is locally parametrized by one-parameter family of AdS-horocycle at any point. Especially, we call an AdS-horocyclic surface a TAdS-horocyclic surface or a $S A d S$ horocyclic surface if it is locally parametrized by one-parameter family of TAdS-horocycle or SAdS-horocycle respectively. Eventually we have shown the following theorem.

Theorem 3.6 If $M \subset H_{1}^{3}$ is an umbilically free AdS-horo-flat surfce, it is an AdS-horocyclic surface. Moreover, each AdS-horocycle is the line of curvature with the vanishing AdS-null principle curvature.

Proof. We take the flat TAdS-horo-surface as an example. So that the first part of the theorem is a corollary of Proposition 3.3. For the second part, we assume that $M=\boldsymbol{X}(U)$, both the $u$-curve and $v$-curve are the lines of curvature which satisfy $\mathbb{G}_{n u}(u, v)=0, \mathbb{G}_{n v}(u, v)=$ $-k(u, v) \boldsymbol{X}_{v}(u, v)$ and $u$-curve is a timelike curve. We now consider the parametrization

$$
\overline{\boldsymbol{X}}(s, v)=\boldsymbol{X}(0, v)+s \frac{\boldsymbol{X}_{u}(0, v)}{\left\|\boldsymbol{X}_{u}(0, v)\right\|}-\frac{s^{2}}{2} \mathbb{G}_{n}(0, v)
$$

of $M=\boldsymbol{X}(U)$. By a straightforward calculation, we have

$$
\overline{\boldsymbol{X}}_{s}(s, v)=\frac{\boldsymbol{X}_{u}(0, v)}{\left\|\boldsymbol{X}_{u}(0, v)\right\|}-s \mathbb{G}_{n}(0, v),
$$




$$
\overline{\boldsymbol{X}}_{v}(s, v)=\boldsymbol{X}_{v}(0, v)+s\left(\frac{\boldsymbol{X}_{u}(0, v)}{\left\|\boldsymbol{X}_{u}(0, v)\right\|}\right)_{v}-\frac{s^{2}}{2} \mathbb{G}_{n v}(0, v) .
$$

Since $\left\langle\mathbb{G}_{n}(0, v), \boldsymbol{X}_{u}(0, v)\right\rangle=0$, we have $\left\langle\mathbb{G}_{n v}(0, v), \boldsymbol{X}_{u}(0, v)\right\rangle+\left\langle\mathbb{G}_{n}(0, v), \boldsymbol{X}_{u v}(0, v)\right\rangle=0$. It follows that $\left\langle\mathbb{G}_{n}(0, v), \boldsymbol{X}_{u v}(0, v)\right\rangle=0$. Since $\mathbb{G}_{n}(0, v)$ is the null normal vector of $M=\boldsymbol{X}(U)$ at $\boldsymbol{X}(0, v)$, we have $\left\langle\mathbb{G}_{n}(0, v), \overline{\boldsymbol{X}}_{s}(s, v)\right\rangle=\left\langle\mathbb{G}_{n}(0, v), \overline{\boldsymbol{X}}_{v}(s, v)\right\rangle=0$. This means that $\mathbb{G}_{n}(0, v)$ is the null normal of $M=\boldsymbol{X}(U)$ at $\overline{\boldsymbol{X}}(s, v)$. So that we have the null normal $\mathbb{G}_{n}$ which is constant along the $s$-curve. Since the $s$-curve is a TAdS-horocycle, it is the line of curvature with vanishing AdS-null principal curvature.

\section{AdS-horocyclic surfaces}

In this section we study general properties of AdS-horocyclic surfaces. Let $\boldsymbol{a}_{i}: I \longrightarrow H_{1}^{3}$ $(i=0,1)$ and $\boldsymbol{a}_{3}: I \longrightarrow S_{2}^{3}$ be smooth mappings from an open interval $I$ with $\left\langle\boldsymbol{a}_{i}(t), \boldsymbol{a}_{3}(t)\right\rangle=0$ and $\left\langle\boldsymbol{a}_{1}(t), \boldsymbol{a}_{2}(t)\right\rangle=0$. We define a unit spacelike vector $\boldsymbol{a}_{4}(t)=\boldsymbol{a}_{1}(t) \wedge \boldsymbol{a}_{2}(t) \wedge \boldsymbol{a}_{3}(t)$. Therefore, we have a pseudo-orthonormal frame $\left\{\boldsymbol{a}_{1}, \boldsymbol{a}_{2}, \boldsymbol{a}_{3}, \boldsymbol{a}_{4}\right\}$ of $\mathbb{R}_{2}^{4}$. By using this frame, we can define the following basic invariants:

$$
\begin{array}{ll}
c_{1}(t)=-\left\langle\boldsymbol{a}_{1}^{\prime}(t), \boldsymbol{a}_{2}(t)\right\rangle=\left\langle\boldsymbol{a}_{1}(t), \boldsymbol{a}_{2}^{\prime}(t)\right\rangle, & c_{4}(t)=\left\langle\boldsymbol{a}_{2}^{\prime}(t), \boldsymbol{a}_{3}(t)\right\rangle=-\left\langle\boldsymbol{a}_{2}(t), \boldsymbol{a}_{3}^{\prime}(t)\right\rangle \\
c_{2}(t)=\left\langle\boldsymbol{a}_{1}^{\prime}(t), \boldsymbol{a}_{3}(t)\right\rangle=-\left\langle\boldsymbol{a}_{1}(t), \boldsymbol{a}_{3}^{\prime}(t)\right\rangle, & c_{5}(t)=\left\langle\boldsymbol{a}_{2}^{\prime}(t), \boldsymbol{a}_{4}(t)\right\rangle=-\left\langle\boldsymbol{a}_{2}(t), \boldsymbol{a}_{4}^{\prime}(t)\right\rangle \\
c_{3}(t)=\left\langle\boldsymbol{a}_{1}^{\prime}(t), \boldsymbol{a}_{4}(t)\right\rangle=-\left\langle\boldsymbol{a}_{1}(t), \boldsymbol{a}_{4}^{\prime}(t)\right\rangle, & c_{6}(t)=\left\langle\boldsymbol{a}_{3}^{\prime}(t), \boldsymbol{a}_{4}(t)\right\rangle=-\left\langle\boldsymbol{a}_{3}(t), \boldsymbol{a}_{4}^{\prime}(t)\right\rangle
\end{array}
$$

Then we have the following fundamental differential equations:

$$
\left\{\begin{array}{l}
\boldsymbol{a}_{1}^{\prime}(t)=c_{1}(t) \boldsymbol{a}_{2}(t)+c_{2}(t) \boldsymbol{a}_{3}(t)+c_{3}(t) \boldsymbol{a}_{4}(t) \\
\boldsymbol{a}_{2}^{\prime}(t)=-c_{1}(t) \boldsymbol{a}_{1}(t)+c_{4}(t) \boldsymbol{a}_{3}(t)+c_{5}(t) \boldsymbol{a}_{4}(t) \\
\boldsymbol{a}_{3}^{\prime}(t)=c_{2}(t) \boldsymbol{a}_{1}(t)+c_{4}(t) \boldsymbol{a}_{2}(t)+c_{6}(t) \boldsymbol{a}_{4}(t) \\
\boldsymbol{a}_{4}^{\prime}(t)=c_{3}(t) \boldsymbol{a}_{1}(t)+c_{5}(t) \boldsymbol{a}_{2}(t)-c_{6}(t) \boldsymbol{a}_{3}(t)
\end{array}\right.
$$

We can rewrite this equations as follows:

$$
A^{\prime}(t)=C(t) A(t)
$$

where

$$
A=\left(\begin{array}{l}
\boldsymbol{a}_{1}(t) \\
\boldsymbol{a}_{2}(t) \\
\boldsymbol{a}_{3}(t) \\
\boldsymbol{a}_{4}(t)
\end{array}\right), \quad C=\left(\begin{array}{cccc}
0 & c_{1}(t) & c_{2}(t) & c_{3}(t) \\
-c_{1}(t) & 0 & c_{4}(t) & c_{5}(t) \\
c_{2}(t) & c_{4}(t) & 0 & c_{6}(t) \\
c_{3}(t) & c_{5}(t) & -c_{6}(t) & 0
\end{array}\right) .
$$

We remark that $A(t) \in S O_{o}(2,2)$ and $C(t) \in \mathfrak{s o}(2,2)$, where $\mathfrak{s o}(2,2)$ is the Lie algebra of the Lorentzian group $S O_{o}(2,2)$.

We define a mapping $F_{\left(a_{0}, a_{2}, a_{1}, a_{3}\right)}: \mathbb{R} \times I \longrightarrow H_{1}^{3}$ by

$$
F_{\left(a_{1}, a_{2}, a_{3}, a_{4}\right)}(s, t)=\boldsymbol{a}_{1}(t)+s\left[\frac{1-\varepsilon}{2} \boldsymbol{a}_{2}(t)+\frac{1+\varepsilon}{2} \boldsymbol{a}_{4}(t)\right]+\varepsilon \frac{s^{2}}{2} \boldsymbol{g}(t),
$$

where $\varepsilon= \pm 1, \boldsymbol{g}(t)=\boldsymbol{a}_{1}(t)+\boldsymbol{a}_{3}(t)$. In the case when $\varepsilon=-1$, we denote $F_{\left(a_{1}, a_{2}, a_{3}, a_{4}\right)}(s, t)$ by

$$
F_{\left(a_{1}, a_{2}, a_{3}\right)}^{T}(s, t)=\boldsymbol{a}_{1}(t)+s \boldsymbol{a}_{2}(t)-\frac{s^{2}}{2} \boldsymbol{g}(t) .
$$


When $\varepsilon=1$, we denote $F_{\left(a_{1}, a_{2}, a_{3}, a_{4}\right)}(s, t)$ by

$$
F_{\left(a_{1}, a_{3}, a_{4}\right)}^{S}(s, t)=\boldsymbol{a}_{1}(t)+s \boldsymbol{a}_{4}(t)+\frac{s^{2}}{2} \boldsymbol{g}(t) .
$$

By Proposition 3.3 and Proposition 3.5, we have a TAdS-horocycle $F_{\left(a_{1}, a_{2}, a_{3}\right)}^{T}\left(s, t_{0}\right)$ and a SAdShorocycle $F_{\left(a_{1}, a_{3}, a_{4}\right)}^{S}\left(s, t_{0}\right)$ respectively for any fixed $t=t_{0}$. We call $F_{\left(a_{1}, a_{2}, a_{3}, a_{4}\right)}$ (or it's image) an AdS-horocyclic surface. Each TAdS-horocycle $F_{\left(a_{1}, a_{2}, a_{3}\right)}^{T}\left(s, t_{0}\right)$ or SAdS-horocycle $F_{\left(a_{1}, a_{3}, a_{4}\right)}^{S}\left(s, t_{0}\right)$ is called a generating AdS-horocycle.

If $\left\{\boldsymbol{a}_{1}(t), \boldsymbol{a}_{2}(t), \boldsymbol{a}_{3}(t), \boldsymbol{a}_{4}(t)\right\}$ is a pseudo-orthonormal frame field as the above, the $4 \times$ 4-matrix determined by the frame defines a smooth curve $A: I \longrightarrow S O_{o}(2,2)$. So that we have shown that $A^{\prime}(t)=C(t) A(t)$. For the converse, if $A: I \longrightarrow S O_{o}(2,2)$ is a smooth curve, then we can show that $A^{\prime}(t) A(t)^{-1} \in \mathfrak{s o}(2,2)$. Moreover, for any smooth curve $C: I \longrightarrow \mathfrak{s o}(2,2)$, we apply the existence theorem on the linear systems of ODEs $(*)$, so that there exists a unique curve $A: I \longrightarrow S O_{o}(2,2)$ such that $C(t)=A^{\prime}(t) A(t)^{-1}$ with an initial data $A\left(t_{0}\right) \in S O_{o}(2,2)$. Therefore, a smooth curve $C: I \longrightarrow \mathfrak{s o}(2,2)$ might be identified with an AdS-horocyclic surface in $H_{1}^{3}$. Moreover, if $C: I \longrightarrow \mathfrak{s o}(2,2)$ is a smooth curve with $C(t)=A^{\prime}(t) A(t)^{-1}$ and $B \in S O_{o}(2,2)$ then we have $C(t)=(A(t) B)^{\prime}(A(t) B)^{-1}$. This means that the curve $C: I \longrightarrow \mathfrak{s o}(2,2)$ is a Lorentz invariant of the pseudo-orthonormal frame $\left\{\boldsymbol{a}_{1}(t), \boldsymbol{a}_{2}(t), \boldsymbol{a}_{3}(t), \boldsymbol{a}_{4}(t)\right\}$, so that it is a Lorentz invariant of the corresponding AdS-horocyclic surface. We write $F_{A}$ instead of $F_{\left(a_{0}, a_{1}, a_{2}, a_{3}\right)}$.

Let $C^{\infty}(I, \mathfrak{s o}(2,2))$ be the space of smooth curves into $\left.\mathfrak{s o}(2,2)\right)$ equipped with Whitney $C^{\infty}$-topology. By the above arguments, we may regard $C^{\infty}(I, \mathfrak{s o}(2,2))$ as the space of AdS-horocyclic surfaces, where $I$ is an open interval or the unit circle. We assume that $C_{T}^{\infty}(I, \mathfrak{s o}(2,2))$ is the space of TAdS-horocyclic surfaces and $C_{S}^{\infty}(I, \mathfrak{s o}(2,2))$ is the space of SAdS-horocyclic surfaces, then we have that $C^{\infty}(I, \mathfrak{s o}(2,2))=C_{T}^{\infty}(I, \mathfrak{s o}(2,2)) \cup C_{S}^{\infty}(I, \mathfrak{s o}(2,2))$.

\subsection{Singularities of TAdS-horocyclic surfaces}

We firstly consider the geometric properties of TAdS-horocyclic surfaces. Let $F_{\left(a_{1}, a_{2}, a_{3}\right)}^{T}: \mathbb{R} \times$ $I \longrightarrow H_{1}^{3}$ be a TAdS-horocyclic surface defined by

$$
F_{\left(a_{1}, a_{2}, a_{3}\right)}^{T}(s, t)=\boldsymbol{a}_{1}(t)+s \boldsymbol{a}_{2}(t)-\frac{s^{2}}{2} \boldsymbol{g}(t)
$$

We consider a parameter transformation

$$
S=s(t)-s, T=t
$$

for any smooth function $s(t)$. We define $\bar{A}(t)=\left(\overline{\boldsymbol{a}}_{1}(t), \overline{\boldsymbol{a}}_{2}(t), \overline{\boldsymbol{a}}_{3}(t), \overline{\boldsymbol{a}}_{4}(t)\right)^{t}$ by

$$
\begin{gathered}
\overline{\boldsymbol{a}}_{1}(t)=\boldsymbol{a}_{1}(t)+s(t) \boldsymbol{a}_{2}(t)-\frac{s^{2}(t)}{2} \boldsymbol{g}(t), \quad \overline{\boldsymbol{a}}_{2}(t)=-\boldsymbol{a}_{2}(t)+s(t) \boldsymbol{g}(t), \\
\overline{\boldsymbol{a}}_{3}(t)=\boldsymbol{g}(t)-\overline{\boldsymbol{a}}_{1}(t), \quad \overline{\boldsymbol{a}}_{4}(t)=\overline{\boldsymbol{a}}_{1}(t) \wedge \overline{\boldsymbol{a}}_{2}(t) \wedge \overline{\boldsymbol{a}}_{3}(t)=-\boldsymbol{a}_{4}(t),
\end{gathered}
$$

where $(A)^{t}$ denote the transposed matrix. It is obviously that

$$
\bar{A}(t) \in S O_{o}(2,2), \quad F_{\left(a_{1}, a_{2}, a_{3}\right)}^{T}(s, t)=F_{\left(\bar{a}_{1}, \bar{a}_{2}, \bar{a}_{3}\right)}^{T}(S, T) .
$$

It follows that $\overline{\boldsymbol{a}}_{1}(t)$ is the curve on the TAdS-horocyclic surface $F_{\left(\bar{a}_{1}, \bar{a}_{2}, \bar{a}_{3}\right)}^{T}(S, T)$ defined by the equation $S=0$. We call the above parameter transformation an T-adapted parameter 
transformation. By straightforward calculations, we have

$$
\left\{\begin{array}{l}
\bar{c}_{1}(t)=-c_{1}(t)-s(t) c_{2}(t)-s^{2}(t) c_{14}(t) / 2-s^{\prime}(t) \\
\bar{c}_{2}(t)=c_{2}(t)+s(t) c_{14}(t) \\
\bar{c}_{3}(t)=-c_{3}(t)-s(t) c_{5}(t)+s^{2}(t) c_{36}(t) / 2 \\
\bar{c}_{4}(t)=-c_{4}(t)+s(t) c_{2}(t)+s^{2}(t) c_{14}(t) / 2+s^{\prime}(t) \\
\bar{c}_{5}(t)=c_{5}(t)-s(t) c_{36}(t) \\
\bar{c}_{6}(t)=-c_{6}(t)+s(t) c_{5}(t)-s^{2}(t) c_{36}(t) / 2
\end{array}\right.
$$

where we denote $c_{1}(t)+c_{4}(t)=c_{14}(t)$ and $c_{3}(t)+c_{6}(t)=c_{36}(t)$ for simply.

It follows that we have

$$
\bar{c}_{1}(t)+\bar{c}_{4}(t)=-c_{14}(t), \quad \bar{c}_{3}(t)+\bar{c}_{6}(t)=-c_{36}(t), \quad \bar{c}_{1}+\bar{c}_{4}(t)=\bar{c}_{2}(t)=0 \Leftrightarrow c_{14}(t)=c_{2}(t)=0
$$

Proposition 4.1 Let $F_{\left(a_{1}, a_{2}, a_{3}\right)}^{T}$ be a parameterization of a TAdS-horocyclic surface of the form

$$
F_{\left(a_{1}, a_{2}, a_{3}\right)}^{T}(s, t)=\boldsymbol{a}_{1}(t)+s \boldsymbol{a}_{2}(t)-\frac{s^{2}}{2} \boldsymbol{g}(t)
$$

such that $c_{14}(t)$ never vanishes. Then the image of $F_{\left(a_{1}, a_{2}, a_{3}\right)}^{T}$ has a reparametrization of the form

$$
F_{\left(\bar{a}_{1}, \bar{a}_{2}, \bar{a}_{3}\right)}^{T}(S, T)=\overline{\boldsymbol{a}}_{1}(t)+S \overline{\boldsymbol{a}}_{2}(t)-\frac{S^{2}}{2} \boldsymbol{g}(T),
$$

where $\bar{c}_{2}(t)=0$ and $s(t)=-c_{2}(t) / c_{14}(t)$.

Proof. We define $\overline{\boldsymbol{a}}_{1}, \overline{\boldsymbol{a}}_{2}, \overline{\boldsymbol{a}}_{3}$ as the above notations. By a straightforward calculation, we have $\bar{c}_{2}(t)=\left\langle\overline{\boldsymbol{a}}_{1}^{\prime}(t), \overline{\boldsymbol{a}}_{3}(t)\right\rangle=\left\langle\overline{\boldsymbol{a}}_{1}^{\prime}(t), \boldsymbol{g}(t)\right\rangle$. We define $s(t)$ by $s(t)=-c_{2}(t) / c_{14}(t)$, then we have $\bar{c}_{2}(t)=0$.

We define $F_{\left(\bar{a}_{1}, \bar{a}_{2}, \bar{a}_{3}\right)}^{T}(S, T)=F_{\left(\bar{a}_{1}, \bar{a}_{2}, \bar{a}_{3}\right)}^{T}(s(t)-s, t)$. Then we have

$$
F_{\left(\bar{a}_{1}, \bar{a}_{2}, \bar{a}_{3}\right)}^{T}(S, T)=F_{\left(a_{1}, a_{2}, a_{3}\right)}^{T}(s, t) .
$$

Therefore, $F_{\left(\bar{a}_{1}, \bar{a}_{2}, \bar{a}_{3}\right)}^{T}$ and $F_{\left(a_{1}, a_{2}, a_{3}\right)}^{T}$ have the same image.

We call the curve $\overline{\boldsymbol{a}}_{1}$ the striction curve of $F_{\left(a_{1}, a_{2}, a_{3}\right)}^{T}$ if $\left\langle\overline{\boldsymbol{a}}_{1}^{\prime}(t), \overline{\boldsymbol{a}}_{3}(t)\right\rangle=0$. By the above proposition, we have the unique striction curve under the condition that $c_{14}(t) \neq 0$, then it is given by the equation $S=0$ after the above T-adapted parameter transformation. In the case when $c_{14}(t)=0$, there exist striction curve iff $c_{2}(t)=0$. Therefore, when $c_{14}(t) \neq 0$ or $c_{14}(t)=c_{2}(t)=0$, we may assume that $\boldsymbol{a}_{1}(t)$ is the striction curve of $F_{\left(a_{1}, a_{2}, a_{3}\right)}^{T}(s, t)$ which is given by $s=0$ by an T-adapted parameter transformation.

We now consider the singularities of TAdS-horocyclic surfaces. By a straightforward calculation, we have

$$
\begin{aligned}
\frac{\partial F_{\left(a_{1}, a_{2}, a_{3}\right)}^{T}}{\partial s}= & \boldsymbol{a}_{2}(t)-s \boldsymbol{g}(t)=-s \boldsymbol{a}_{1}(t)+\boldsymbol{a}_{2}(t)-s \boldsymbol{a}_{3}(t) \\
\frac{\partial F_{\left(a_{1}, a_{2}, a_{3}\right)}^{T}=}{\partial t}= & -\left(s c_{1}(t)+\frac{s^{2}}{2} c_{2}(t)\right) \boldsymbol{a}_{1}(t)+\left(c_{1}(t)-\frac{s^{2}}{2} c_{14}(t)\right) \boldsymbol{a}_{2}(t) \\
& \quad+\left[\left(1-\frac{s^{2}}{2}\right) c_{2}(t)+s c_{4}(t)\right] \boldsymbol{a}_{3}(t)+\left(c_{3}(t)-\frac{s^{2}}{2} c_{36}(t)+s c_{5}(t)\right) \boldsymbol{a}_{4}(t) .
\end{aligned}
$$

It follows that $(s, t)$ is a singular point of $F_{\left(a_{1}, a_{2}, a_{3}\right)}^{T}$ if and only if $\bar{c}_{2}(t)=0$ and $\bar{c}_{3}(t)=0$ for the T-adapted parameter transformation $S=s(t)-s, T=t$. The above condition is equivalent to the condition that $S=0$ is a singular point. Then we have the following proposition. 
Proposition 4.2 Let $F_{\left(a_{1}, a_{2}, a_{3}\right)}^{T}$ be a TAdS-horocyclic surface with the striction curve $\boldsymbol{a}_{1}$ and $c_{14}(t) \neq 0$. If $x_{0}=F_{\left(a_{1}, a_{2}, a_{3}\right)}^{T}\left(s_{0}, t_{0}\right)$ is a singular value of the TAdS-horocyclic surface $F_{\left(a_{1}, a_{2}, a_{3}\right)}^{T}$, then $s_{0}=0$, i.e., $x_{0}$ is located on the image of $\boldsymbol{a}_{1}$ such that $\boldsymbol{a}_{2}\left(t_{0}\right)$ is tangent to $\boldsymbol{a}_{1}$ at $t_{0}$ under the condition $\boldsymbol{a}_{1}^{\prime}\left(t_{0}\right) \neq 0$.

Proof. If $\boldsymbol{a}_{1}^{\prime}\left(t_{0}\right)=0$, then $F_{\left(a_{1}, a_{2}, a_{3}\right)}^{T}\left(0, t_{0}\right)$ is only singular value on $F_{\left(a_{1}, a_{2}, a_{3}\right)}^{T}\left(s, t_{0}\right)$. Therefore we assume that $\boldsymbol{a}_{1}$ is a unit speed timelike curve. Since $c_{2} \equiv 0$ and $c_{14} \neq 0$, it follows that if $\left(s_{0}, t_{0}\right)$ is a singular point of $F_{\left(a_{1}, a_{2}, a_{3}\right)}^{T}$, then $s_{0}=0$ and hence $x_{0}$ is located on the image of $\boldsymbol{a}_{1}$. Since singular set is given by the set $\left\{\left(0, t_{0}\right) \mid c_{2}\left(t_{0}\right)=c_{3}\left(t_{0}\right)=0\right\}, \boldsymbol{a}_{1}^{\prime}\left(t_{0}\right)$ is pseudo-orthogonal to $\boldsymbol{a}_{1}\left(t_{0}\right), \boldsymbol{a}_{3}\left(t_{0}\right), \boldsymbol{a}_{4}\left(t_{0}\right)$. Therefore it is tangent to $\boldsymbol{a}_{2}\left(t_{0}\right)$.

We call the kernel direction of $d F_{\left(a_{1}, a_{2}, a_{3}\right)}^{T}$ the null direction or the null vector field and denote it by $\eta^{T}$. Then it holds that

$$
\begin{aligned}
S\left(F_{\left(a_{1}, a_{2}, a_{3}\right)}^{T}\right) & =\left\{(s, t) \mid c_{2}(t)+s c_{14}(t)=0,-c_{3}(t)-s c_{5}(t)+s^{2} c_{36}(t) / 2=0\right\} \\
\eta(s, t) & =\operatorname{ker} d F_{\left(a_{1}, a_{2}, a_{3}\right)}^{T}(s, t)=\left(c_{1}(t)-\frac{s^{2}}{2} c_{14}(t)\right) \frac{\partial}{\partial s}-\frac{\partial}{\partial t}
\end{aligned}
$$

where, $S\left(F_{\left(a_{1}, a_{2}, a_{3}\right)}^{T}\right)$ is the singular set of $F_{\left(a_{1}, a_{2}, a_{3}\right)}^{T}$. Then we have the following:

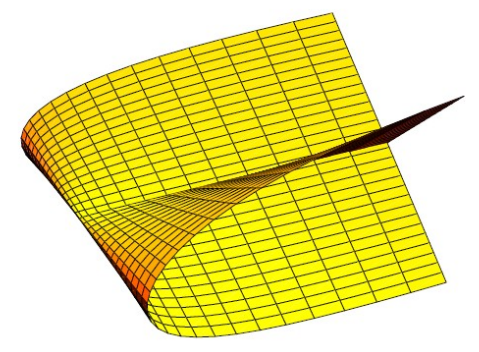

Figure 1: cross cap

Theorem 4.3 A point $\left(s_{0}, t_{0}\right) \in S\left(F_{\left(a_{1}, a_{2}, a_{3}\right)}^{T}\right)$ is a cross cap if and only if $c_{2}\left(t_{0}\right)+s_{0} c_{14}\left(t_{0}\right)=0$, $-c_{3}\left(t_{0}\right)-s_{0} c_{5}\left(t_{0}\right)+s_{0}^{2} c_{36}\left(t_{0}\right) / 2=0$ and $C R^{T}\left(s_{0}, t_{0}\right) \neq 0$, where

$$
C R^{T}(s, t)=\left(-s c_{36}(t)+c_{5}(t)\right)\left(c_{2}^{\prime}(t)+s c_{14}^{\prime}(t)\right)+c_{14}(t)\left(-2 c_{3}^{\prime}(t)-2 s c_{5}^{\prime}(t)+s^{2} c_{36}^{\prime}(t)\right) .
$$

If $c_{14} c_{2} \neq 0$ at $t_{0}$, then these conditions are equivalent to

$$
s_{0}=\left(-c_{2} / c_{14}\right)\left(t_{0}\right), \quad \kappa^{T}\left(t_{0}\right)=0, \quad\left(\kappa^{T}\right)^{\prime}\left(t_{0}\right) \neq 0, \quad\left(\kappa^{T}(t)=\left(-2 c_{14}^{2} c_{3}+c_{2}^{2} c_{36}+2 c_{14} c_{2} c_{5}\right)(t)\right) .
$$

Here, a map germ $f:\left(\mathbb{R}^{2}, \boldsymbol{a}\right) \longrightarrow\left(\mathbb{R}^{3}, \boldsymbol{b}\right)$ is called a cross cap if it is $\mathcal{A}$-equivalent to the germ $\left(u_{1}^{2}, u_{2}, u_{1} u_{2}\right)$ (cf., Figure 1)

Proof. For simply, we denote $F_{\left(a_{1}, a_{2}, a_{3}\right)}^{T}$ by $F$ in this proof. Since the null vector (the kernel of $d F)$ is given by $\eta=\left(c_{1}-s^{2} c_{14} / 2\right)(\partial / \partial s)-\partial / \partial t$, by Whitney's theorem [29, Theorem 1], if

$$
\operatorname{det}\left(F, F_{s}, \eta F_{s}, \eta \eta F\right) \neq 0
$$

then $F$ at the point is a cross cap. By a very long calculation, we have

$$
\operatorname{det}\left(F, F_{s}, \eta F_{s}, \eta \eta F\right)=\left(c_{5}-s c_{36}\right)\left(-2 c_{3}-2 s c_{5}+s^{2} c_{36}\right)\left(2 c_{6}-2 s c_{5}+s^{2} c_{36}\right)+C R\left(s_{0}, t_{0}\right)
$$


The first term of the right-hand side of the above equation is zero since $\left(s_{0}, t_{0}\right)$ is a singular point. Thus we have the first part of the theorem. If $c_{14} c_{2} \neq 0$ at $t_{0}$, then the conditions $c_{2}\left(t_{0}\right)+s_{0} c_{14}\left(t_{0}\right)=0$ and $-c_{3}\left(t_{0}\right)-s_{0} c_{5}\left(t_{0}\right)+s_{0}^{2} c_{36}\left(t_{0}\right) / 2=0$ are equivalent to $s_{0}=\left(-c_{2} / c_{14}\right)\left(t_{0}\right)$ and $\kappa^{T}\left(t_{0}\right)=0$. Substituting $s=-c_{2} / c_{14}$ and $c_{36}=2\left(c_{14}^{2} c_{3}-c_{14} c_{2} c_{5}\right) / c_{2}^{2}$ to $C R^{T} \neq 0$, we have $\left(\kappa^{T}\right)^{\prime} \neq 0$.

We remark that if we consider $\boldsymbol{a}_{1}$ as striction curve, then $c_{2} \equiv 0$, so that $s\left(t_{0}\right)=0$. Moreover, we have $c_{3}\left(t_{0}\right)=0$. Since $c_{14}\left(t_{0}\right) \neq 0$, the condition for cross cap on the striction curve is $c_{3}\left(t_{0}\right)=0, c_{3}^{\prime}\left(t_{0}\right) \neq 0$. This condition is a generic condition in $C_{T}^{\infty}(I, \mathfrak{s}(2,2))$. Therefore, we have the following theorem.

Theorem 4.4 There exists an open dense subset $\mathcal{O} \subset C_{T}^{\infty}(I, \mathfrak{s o}(2,2))$ such that for any $C \in \mathcal{O}$, the germ of the TAdS-horocyclic surface $F_{\left(a_{1}, a_{2}, a_{3}\right)}^{T}$ at a singular point is diffeomorphic to the cross cap.

\subsection{Singularities of SAdS-horocyclic surfaces}

In this section, we investigate the generic singularities of SAdS-horocyclic surfaces by a similar way as those of TAdS-horocyclic surfaces. Let $F_{\left(a_{1}, a_{3}, a_{4}\right)}^{S}: \mathbb{R} \times I \longrightarrow H_{1}^{3}$ be a STAdS-horocyclic surfaces defined by

$$
F_{\left(a_{1}, a_{3}, a_{4}\right)}^{S}(s, t)=\boldsymbol{a}_{1}(t)+s \boldsymbol{a}_{4}(t)+\frac{s^{2}}{2} \boldsymbol{g}(t) .
$$

We consider another parameter transformation $S=s-s(t), T=t$ for any smooth function $s(t)$. We define $\widehat{A}(t)=\left(\widehat{\boldsymbol{a}}_{1}(t), \widehat{\boldsymbol{a}}_{2}(t), \widehat{\boldsymbol{a}}_{3}(t), \widehat{\boldsymbol{a}}_{4}(t)\right)^{t}$ by

$$
\begin{gathered}
\widehat{\boldsymbol{a}}_{1}(t)=\boldsymbol{a}_{1}(t)+s(t) \boldsymbol{a}_{4}(t)+\frac{s^{2}(t)}{2} \boldsymbol{g}(t), \quad \widehat{\boldsymbol{a}}_{2}(t)=\boldsymbol{a}_{2}(t), \\
\widehat{\boldsymbol{a}}_{3}(t)=\boldsymbol{g}(t)-\widehat{\boldsymbol{a}}_{1}(t), \quad \widehat{\boldsymbol{a}}_{4}(t)=\boldsymbol{a}_{4}(t)+s(t) \boldsymbol{g}(t),
\end{gathered}
$$

It is obviously that

$$
\widehat{A}(t) \in S O_{o}(2,2) \quad \text { and } \quad F_{\left(a_{1}, a_{3}, a_{4}\right)}^{S}(s, t)=F_{\left(\hat{a}_{1}, \hat{a}_{3}, \hat{a}_{4}\right)}^{S}(S, T) .
$$

It follows that $\widehat{\boldsymbol{a}}_{1}(t)$ is the curve on the SAdS-horocyclic surface $F_{\left(\hat{a}_{1}, \hat{a}_{3}, \hat{a}_{4}\right)}^{S}(S, T)$ defined by the equation $S=0$. We also call the above parameter transformation an $S$-adapted parameter transformation. By straightforward calculations, we have

$$
\left\{\begin{array}{l}
\widehat{c}_{1}(t)=c_{1}(t)+s(t) c_{5}(t)+s^{2}(t) c_{14}(t) / 2 \\
\widehat{c}_{2}(t)=c_{2}(t)-s(t) c_{36}(t) \\
\widehat{c}_{3}(t)=c_{3}(t)+s(t) c_{2}(t)-s^{2}(t) c_{36}(t) / 2+s^{\prime}(t) \\
\widehat{c}_{4}(t)=c_{4}(t)-s(t) c_{5}(t)-s^{2}(t) c_{14}(t) / 2 \\
\widehat{c}_{5}(t)=c_{5}(t)+s(t) c_{14}(t) \\
\widehat{c}_{6}(t)=c_{6}(t)-s(t) c_{2}(t)+s^{2}(t) c_{36}(t) / 2-s^{\prime}(t) .
\end{array}\right.
$$

It follows that we have

$$
\widehat{c}_{1}(t)+\widehat{c}_{4}(t)=c_{14}(t), \quad \widehat{c}_{3}(t)+\widehat{c}_{6}(t)=c_{36}(t), \quad \widehat{c}_{2}(t)=\widehat{c}_{3}+\widehat{c}_{6}(t)=0 \Leftrightarrow c_{2}(t)=c_{36}(t)=0
$$

By almost the same way as Proposition 4.1, we have the following:

Proposition 4.5 Let $F_{\left(a_{1}, a_{3}, a_{4}\right)}^{S}$ be a parameterization of a SAdS-horocyclic surface of the form

$$
F_{\left(a_{1}, a_{3}, a_{4}\right)}^{S}(s, t)=\boldsymbol{a}_{1}(t)+s \boldsymbol{a}_{4}(t)+\frac{s^{2}}{2} \boldsymbol{g}(t)
$$


such that $c_{36}(t)$ never vanishes. Then the image of $F_{\left(a_{1}, a_{3}, a_{4}\right)}^{S}$ has a reparametrization of the form

$$
F_{\left(\hat{a}_{1}, \hat{a}_{3}, \hat{a}_{4}\right)}^{S}=\widehat{\boldsymbol{a}}_{1}(t)+S \widehat{\boldsymbol{a}}_{4}(t)+\frac{S^{2}}{2} \boldsymbol{g}(T),
$$

where $\widehat{c}_{2}(t)=0$ and $s(t)=\frac{c_{2}(t)}{c_{36}(t)}$.

We call the curve $\widehat{\boldsymbol{a}}_{1}$ the striction curve of $F_{\left(a_{1}, a_{3}, a_{4}\right)}^{S}$ if $\left\langle\widehat{\boldsymbol{a}}_{1}^{\prime}(t), \widehat{\boldsymbol{a}}_{2}(t)\right\rangle=0$. By the above proposition, we have the unique striction curve under the condition that $c_{36}(t) \neq 0$, then it is given by the equation $S=0$ after the above $S$-adapted parameter transformation. In the case when $c_{36}(t)=0$, there exist striction curve iff $c_{2}(t)=0$. Therefore, when $c_{36}(t) \neq 0$ or $c_{2}(t)=c_{36}(t)=0$, we may assume that $\boldsymbol{a}_{1}(t)$ is the striction curve of $F_{\left(a_{1}, a_{3}, a_{4}\right)}^{S}(s, t)$ which is given by $s=0$ by an S-adapted parameter transformation. By a straightforward calculation, we have

$$
\begin{aligned}
\frac{\partial F_{\left(a_{1}, a_{3}, a_{4}\right)}^{S}}{\partial s}= & \boldsymbol{a}_{4}(t)+s \boldsymbol{g}(t)=s \boldsymbol{a}_{1}(t)+s \boldsymbol{a}_{3}(t)+\boldsymbol{a}_{4}(t) ; \\
\frac{\partial F_{\left(a_{1}, a_{3}, a_{4}\right)}^{S}}{\partial t}= & \boldsymbol{a}_{1}^{\prime}(t)+s \boldsymbol{a}_{4}^{\prime}(t)+\frac{s^{2}}{2} \boldsymbol{g}^{\prime}(t) \\
= & \left(s c_{3}(t)+\frac{s^{2}}{2} c_{2}(t)\right) \boldsymbol{a}_{1}(t)+\left[c_{1}(t)+s c_{5}(t)+\frac{s^{2}}{2} c_{14}(t)\right] \boldsymbol{a}_{2}(t) \\
& +\left[\left(1+\frac{s^{2}}{2}\right) c_{2}(t)-s c_{6}(t)\right] \boldsymbol{a}_{3}(t)+\left[c_{3}(t)+\frac{s^{2}}{2} c_{36}(t)\right] \boldsymbol{a}_{4}(t)
\end{aligned}
$$

It follows that $(s, t)$ is a singular point of $F_{\left(a_{1}, a_{3}, a_{4}\right)}^{S}$ if and only if $\widehat{c}_{1}(t)=0$ and $\widehat{c}_{2}(t)=0$ for the S-adapted parameter transformation $S=s-s(t), T=t$. The above condition is equivalent to the condition that $S=0$ is a singular point. Then we have the following proposition which is similar as the Proposition 4.2.

Proposition 4.6 Let $F_{\left(a_{1}, a_{3}, a_{4}\right)}^{S}$ be a SAdS-horocyclic surface with the striction curve $\boldsymbol{a}_{1}$ and $c_{36}(t) \neq 0$. If $x_{0}=F_{\left(a_{1}, a_{3}, a_{4}\right)}^{S}\left(s_{0}, t_{0}\right)$ is a singular value of the $S A d S$-horocyclic surface $F_{\left(a_{1}, a_{3}, a_{4}\right)}^{S}$, then $s_{0}=0$, i.e., $x_{0}$ is located on the image of $\boldsymbol{a}_{1}$ such that $\boldsymbol{a}_{4}\left(t_{0}\right)$ is tangent to $\boldsymbol{a}_{1}$ at $t_{0}$ under the condition $\boldsymbol{a}_{1}^{\prime}\left(t_{0}\right) \neq 0$.

We call the kernel direction of $d F_{\left(a_{1}, a_{3}, a_{4}\right)}^{S}$ the null direction or the null vector field and denote it by $\zeta$. Then it holds that

$$
\begin{gathered}
S\left(F_{\left(a_{1}, a_{3}, a_{4}\right)}^{S}\right)=\left\{(s, t) \mid \widehat{c}_{1}(t)=0, \widehat{c}_{2}(t)=0\right\} \\
\zeta(s, t)=\operatorname{ker} d F_{\left(a_{1}, a_{3}, a_{4}\right)}^{S}(s ; t)=\left(c_{3}(t)+\frac{s^{2}}{2} c_{36}(t)\right) \frac{\partial}{\partial s}-\frac{\partial}{\partial t},
\end{gathered}
$$

where, $S\left(F_{\left(a_{1}, a_{3}, a_{4}\right)}^{S}\right)$ is the singular set of $F_{\left(a_{1}, a_{3}, a_{4}\right)}^{S}$. Then we have the following theorems:

Theorem 4.7 A point $\left(s_{0}, t_{0}\right) \in S\left(F_{\left(a_{1}, a_{3}, a_{4}\right)}^{S}\right)$ is a cross cap if and only if $\widehat{c}_{1}\left(t_{0}\right)=\widehat{c}_{2}\left(t_{0}\right)=0$ and $C R^{S}\left(s_{0}, t_{0}\right) \neq 0$, where

$$
C R^{S}(s, t)=\left(s c_{14}(t)+c_{5}(t)\right)\left(c_{2}^{\prime}(t)-s c_{36}^{\prime}(t)\right)-c_{36}(t)\left(2 c_{1}^{\prime}(t)+2 s c_{5}^{\prime}(t)+s^{2} c_{14}^{\prime}(t)\right) .
$$

If $c_{36} c_{2} \neq 0$ at $t_{0}$, then these conditions are equivalent to

$$
s_{0}=\left(c_{2} / c_{36}\right)\left(t_{0}\right), \quad \kappa^{S}\left(t_{0}\right)=0, \quad\left(\kappa^{S}\right)^{\prime}\left(t_{0}\right) \neq 0, \quad\left(\kappa^{S}(t)=\left(2 c_{36}^{2} c_{1}+c_{2}^{2} c_{14}+2 c_{36} c_{2} c_{5}\right)(t)\right) .
$$


Theorem 4.8 There exists an open dense subset $\mathcal{O} \subset C_{S}^{\infty}(I, \mathfrak{s o}(2,2))$ such that for any $C \in \mathcal{O}$, the germ of the SAdS-horocyclic surface $F_{\left(a_{1}, a_{3}, a_{4}\right)}^{S}$ at a singular point is diffeomorphic to the cross cap.

According to the Theorems 4.4 and 4.8 , we have the following:

Theorem 4.9 There exists an open dense subset $\mathcal{O} \subset C^{\infty}(I, \mathfrak{s o}(2,2))$ such that for any $C \in \mathcal{O}$, the germ of the AdS-horocyclic surface $F_{\left(a_{1}, a_{2}, a_{3}, a_{4}\right)}$ at a singular point is diffeomorphic to the cross cap.

In the end of this section we consider the geometric meaning of the invariant $\bar{c}_{2}(t)$ and $\widehat{c}_{2}(t)$. By Theorem 3.6, a AdS-horo-flat surfce is an AdS-horocyclic surface $F_{\left(a_{1}, a_{2}, a_{3}, a_{4}\right)}$ with the null normal vector $\boldsymbol{g}(t)$ around a non-umbilical point. In this case, each AdS-horocycle is a line of curvature. However, at an umbilical point, any direction is a principle direction, so that the tangent direction of the AdS-horocycle is also a principle direction. Suppose that $\boldsymbol{g}(t)$ is a null normal vector field on $F_{\left(a_{1}, a_{2}, a_{3}, a_{4}\right)}$. This means that $\widetilde{\mathbb{G}_{n}}(s, t)=\widetilde{\boldsymbol{g}}(t)$. It follows that $\widetilde{\mathbb{G}_{n s}}(s, t)=\widetilde{\boldsymbol{g}}_{s}(t)=0$. Therefore, the tangent component $\Pi_{p} \circ \widetilde{\mathbb{G}_{n s}}(s, t)$ of $\widetilde{\mathbb{G}_{n s}}(s, t)$ is always zero. By Proposition 3.4(2), the AdS-horocyclic surface $F_{\left(a_{1}, a_{2}, a_{3}, a_{4}\right)}$ is a AdS-horo-flat surfce if $\boldsymbol{g}(t)$ is a null normal of the timelike surface. We have shown the following proposition.

Proposition 4.10 An umbilically free AdS-horo-flat surfce is (at least locally) an AdS-horocyclic surface with the null normal vector $\boldsymbol{g}(t)$. Conversely, if $F_{\left(a_{1}, a_{2}, a_{3}, a_{4}\right)}$ is a AdS-horocyclic surface and $\boldsymbol{g}(t)$ is a null normal vector field at any $(s, t)$, then it is a AdS-horo-flat surfce. In this case each AdS-horocycle $F_{\left(a_{1}, a_{2}, a_{3}, a_{4}\right)}\left(s, t_{0}\right)$ is a line of curvature.

Since $\langle\boldsymbol{g}(t), \boldsymbol{g}(t)\rangle=\left\langle\boldsymbol{g}(t), \boldsymbol{g}^{\prime}(t)\right\rangle=\left\langle\boldsymbol{g}(t), \boldsymbol{a}_{2}(t)\right\rangle=0, \boldsymbol{g}(t)$ is a null normal vector at any $(s, t)$ if and only if $\bar{c}_{2}(t)=0$ or $\widehat{c}_{2}(t)=0$. This condition is equivalent to the condition that $c_{2}(t)=c_{14}(t)=0$ or $c_{2}(t)=c_{36}(t)=0$ respectively. By the above proposition, this means that $F_{\left(a_{1}, a_{2}, a_{3}, a_{4}\right)}$ is a AdS-horo-flat surfce if and only if $c_{2}(t)=c_{14}(t)=0$ or $c_{2}(t)=c_{36}(t)=0$. Thus, we say that $F_{\left(a_{1}, a_{2}, a_{3}, a_{4}\right)}$ is a flat AdS-horocyclic surface if $\bar{c}_{2}(t)=0$ or $\widehat{c}_{2}(t)=0$ for any $t$. Especially, we call $F_{\left(a_{1}, a_{2}, a_{3}, a_{4}\right)}$ a flat TAdS-horocyclic surface if $\bar{c}_{2}(t)=0$ or a flat $S A d S$-horocyclic surface if $\widehat{c}_{2}(t)=0$.

\section{$5 \quad$ Flat AdS-horocyclic surfaces}

In this section we study general singularities of flat AdS-horocyclic surfaces. We denote

$$
C=\left(\begin{array}{cccc}
0 & c_{1}(t) & c_{2}(t) & c_{3}(t) \\
-c_{1}(t) & 0 & c_{4}(t) & c_{5}(t) \\
c_{2}(t) & c_{4}(t) & 0 & c_{6}(t) \\
c_{3}(t) & c_{5}(t) & -c_{6}(t) & 0
\end{array}\right) \in \mathfrak{s o}(2,2)
$$

and consider three linear subspaces of $\mathfrak{s o}(2,2)$ defined by

$$
\mathfrak{f a h}(2,2)=\left\{C \in \mathfrak{s o}(2,2) \mid \bar{c}_{2}=0 \text { or } \widehat{c}_{2}=0\right\} .
$$

By the definition of flat AdS-horocyclic surfaces, the space of flat AdS-horocyclic surfaces is defined to be the space $C^{\infty}(I, \mathfrak{f} \mathfrak{a} \mathfrak{h}(2,2))$ equipped with Whitney $C^{\infty}$-topology. We also define the space of flat TAdS-horocyclic surfaces or the space of flat SAdS-horocyclic surfaces by $C^{\infty}\left(I, \mathfrak{f} \mathfrak{a} \mathfrak{h}^{T}(2,2)\right)$ or $C^{\infty}\left(I, \mathfrak{f} \mathfrak{a} \mathfrak{h}^{S}(2,2)\right)$ equipped with Whitney $C^{\infty}$-topology respectively, where

$$
\mathfrak{f a h} \mathfrak{h}^{T}(2,2)=\left\{C \in \mathfrak{s o}(2,2) \mid \bar{c}_{2}=0\right\}, \quad \mathfrak{f a h}^{S}(2,2)=\left\{C \in \mathfrak{s o}(2,2) \mid \widehat{c}_{2}=0\right\}
$$


It is obviously that $\left.\mathfrak{f} \mathfrak{a} \mathfrak{h}(2,2)=\mathfrak{f a h}^{T}(2,2) \cup \mathfrak{f} \mathfrak{a h}\right)^{S}(2,2)$.

\subsection{Singularities of flat TAdS-horocyclic surfaces}

By straightforward calculation, the singular points $(s, t)$ of $F_{\left(a_{1}, a_{2}, a_{3}\right)}^{T}$ satisfy that

$$
\Theta^{T}(s, t)=c_{36}(t) s^{2}-2 c_{5}(t) s-2 c_{3}(t)=0 .
$$

So that the flat TAdS-horocyclic surfaces $F_{\left(a_{1}, a_{2}, a_{3}\right)}^{T}$ has singular points $(s, t)$ if and only if $\Theta^{T}(s, t)=0$ has real roots. Under the condition that $c_{36} \neq 0$, this condition is equivalent to the following condition

$$
\Delta^{T}(t)=c_{5}^{2}(t)+2 c_{3}(t) c_{36}(t) \geq 0 .
$$

Therefore, $\left(s_{0}, t_{0}\right)$ is a singular point of $F_{\left(a_{1}, a_{2}, a_{3}\right)}^{T}$ if the following conditions holds:

(1) $c_{3}\left(t_{0}\right)=0, \Theta^{T}\left(s_{0}, t_{0}\right)=0$

(2) $c_{3}\left(t_{0}\right) \neq 0$ and (i) $c_{36}\left(t_{0}\right)=0, c_{5}\left(t_{0}\right) \neq 0, s_{0}=-\frac{c_{3}\left(t_{0}\right)}{c_{5}\left(t_{0}\right)}$

(ii) $c_{36}\left(t_{0}\right) \neq 0, \Delta^{T}\left(t_{0}\right) \geq 0, \Theta^{T}\left(s_{0}, t_{0}\right)=0$.

If we consider the T-adapted parameter transforation $S=s(t)-s, T=t$, then $S=0, T=t_{0}$ is the singular point and $\bar{c}_{3}\left(t_{0}\right)=0$. So that we may only consider the case (1). We have the following rough classification of singular point under the condition $c_{3}\left(t_{0}\right)=0$ :

(1) if $c_{5}\left(t_{0}\right)=c_{6}\left(t_{0}\right)=0$, then we call $\left(s_{0}, t_{0}\right)$ a TAdS-horocyclic singular point;

(2) if $c_{5}\left(t_{0}\right) \neq 0, c_{6}\left(t_{0}\right)=0$, then we call $\left(s_{0}=0, t_{0}\right)$ a single singular point;

(3) if $c_{5}\left(t_{0}\right)=0, c_{6}\left(t_{0}\right) \neq 0$, then we call $\left(s_{0}=0, t_{0}\right)$ a double singular point;

(4) if $c_{5}\left(t_{0}\right) \neq 0, c_{6}\left(t_{0}\right) \neq 0$, then we call $\left(s_{0}=0, t_{0}\right)$ and $\left(s_{0}=\frac{2 c_{5}\left(t_{0}\right)}{c_{6}\left(t_{0}\right)}, t_{0}\right)$ separated singular points.

We call $F_{\left(a_{1}, a_{2}, a_{3}\right)}^{T}$ a flat TAdS-horocyclic surface with isolated singular point if $c_{3}\left(t_{0}\right)=c_{5}\left(t_{0}\right)=$ $0, s_{0}=0, c_{36}(t) \neq 0, \Delta^{T}(t)<0, \forall t \in I \backslash\left\{t_{0}\right\}$. We now consider a flat TAdS-horocyclic surface with non-isolated singular points and the set of singularities is a union of curves in the parameter space $I \times \mathbb{R}$. In this case, there exist two branches of singularities at most except at the TAdS-horocyclic singular points. However such branches can pass through the TAdS-horocyclic singular points. We assume that one of the branches of the singularities is defined by

$$
\overline{\boldsymbol{a}}_{1}(t)=\boldsymbol{a}_{1}(t)+s(t) \boldsymbol{a}_{2}(t)-\frac{s^{2}(t)}{2} \boldsymbol{g}(t),
$$

where $s=s(t)$ is one of the solutions of the equation $\Theta^{T}(s, t)=0$ for any $t$. In this case we can reparametrize the TAdS-horocyclic surface by $\overline{\boldsymbol{a}}_{2}(t), \overline{\boldsymbol{a}}_{3}(t)$. By the T-adapted parameter transformation, one of the branches of the singularities is located on the curve $S=0$. So that, we may suppose that one of the branches of the singularities is located on $\boldsymbol{a}_{1}(t)$. In this case, such singularities satisfy the condition $c_{3}(t)=0$. Therefore, we assume that $c_{3}(t)=0$ for any $t \in I$. Therefore, $\boldsymbol{a}_{1}^{\prime}(t)$ is parallel to $\boldsymbol{a}_{2}(t)$ if $\boldsymbol{a}_{1}^{\prime}(t) \neq 0$. Moreover, another branches of the singularities is given by the condition $c_{6}(t) s-2 c_{5}(t)=0$. If $c_{6}(t) \neq 0$, we define

$$
\boldsymbol{a}^{\sharp}(t)=\boldsymbol{a}_{1}(t)+s(t) \boldsymbol{a}_{2}(t)-\frac{s^{2}(t)}{2} \boldsymbol{g}(t),
$$

where $s(t)=\frac{2 c_{5}(t)}{c_{6}(t)}$. If $c_{6}(t)=0$, then $c_{5}(t)=0$, it follows that $\widetilde{\boldsymbol{g}}(t)=$ Const $=\widetilde{\boldsymbol{g}}$. In this case $\boldsymbol{a}_{1}$ is a curve located on a AdS-horosphere and the image of $F_{\left(a_{1}, a_{2}, a_{3}\right)}^{T}$ is a subset of the AdS-horosphere. 
We say that $F_{\left(a_{1}, a_{2}, a_{3}\right)}^{T}$ is a flat tangent AdS-horocyclic surface if both $\boldsymbol{a}_{1}$ and $\boldsymbol{a}^{\sharp}$ are not constant or $\boldsymbol{a}_{1}$ is not constant and $c_{6}(t)=0$. In the last case, $F_{\left(a_{1}, a_{2}, a_{3}\right)}^{T}$ is a subset of the AdS-horosphere (a one parameter family of TAdS-horocycles which are tangent to $\boldsymbol{a}_{1}$ on a AdS-horosphere). This condition are equivalent to the conditions that $c_{2}(t)=c_{3}(t)=c_{14}=0$, $c_{1} \neq 0$ and the set of singular points is equal to $s=0$ except the TAdS-horocyclic singular points.

We now define a linear subspaces of $\mathfrak{s o}(2,2)$ by

$$
\mathfrak{f a} \mathfrak{h}_{t}^{T}(2,2)=\left\{C \in \mathfrak{s o}(2,2) \mid c_{2}(t)=c_{3}(t)=c_{14}=0\right\} .
$$

We call space $C^{\infty}\left(I, \mathfrak{f} \mathfrak{a} \mathfrak{h}_{t}^{T}(2,2)\right)$ equipped with Whitney $C^{\infty}$-topology the space of flat tangent TAdS-horocyclic surfaces. In this space, one of the branches of the singularities of the flat TAdS-horocyclic surface is always located on the image of $\boldsymbol{a}_{1}$.

We consider the singular flat TAdS-horocyclic surfaces. By the Thom's jet-transversality theorem $[1,24]$, there exists an open dense set $\mathcal{O} \subset C^{\infty}\left(I, \mathfrak{f} \mathfrak{a} \mathfrak{h}^{T}(2,2)\right)$ such that for any $C \in \mathcal{O}$, it satisfies the condition that $\left(\Delta^{T}(t), \Delta^{T^{\prime}}(t)\right) \neq(0,0)$. If $F_{\left(a_{1}, a_{2}, a_{3}\right)}^{T}$ is flat TAdS-horocyclic surface with an isolated singular point, there exists $t_{0} \in I$ such that $\Delta^{T}\left(t_{0}\right)=\Delta^{T^{\prime}}\left(t_{0}\right)=0$, so that $C \notin \mathcal{O}$. This means that the set of flat TAdS-horocyclic surface with an isolated singular point is not generic in the space of flat TAdS-horocyclic surface. So that the flat tangent TAdS-horocyclic surface is more interesting in the space of the flat TAdS-horocyclic surface.

We now give the classification of singularities of the flat TAdS-horocyclic surface. In this case, we see that the normal vector of $F_{\left(a_{1}, a_{2}, a_{3}\right)}^{T}$ is well-defined and it is $\boldsymbol{g}=\boldsymbol{a}_{1}+\boldsymbol{a}_{3}$. This means that $F_{\left(a_{1}, a_{2}, a_{3}\right)}^{T}$ is a frontal. Moreover we see that

$$
\begin{aligned}
S\left(F_{\left(a_{1}, a_{2}, a_{3}\right)}^{T}\right) & =\left\{(s, t) \mid \lambda^{T}(s, t)=0\right\}, \quad\left(\lambda^{T}(s, t)=c_{3}(t)+s c_{5}(t)-\frac{s^{2} c_{36}(t)}{2}\right), \\
\eta^{T}(s, t) & =\operatorname{ker} d F_{\left(a_{1}, a_{2}, a_{3}\right)}^{T}(s, t)=c_{1}(t) \frac{\partial}{\partial s}-\frac{\partial}{\partial t} .
\end{aligned}
$$

We call $\lambda^{T}$ the T-signed area density function. For $F=F_{\left(a_{1}, a_{2}, a_{3}\right)}^{T}$, by the criteria for singularities of fronts and frontals [6, 21], we have the following.

Theorem 5.1 Let $F$ be a flat TAdS-horocyclic surface and $(s, t) \in S(F)$. Then the following assertions hold.

(1) $F$ at $(s, t)$ is a front if and only if $c_{36} \neq 0$.

(2) $F$ at $(s, t)$ is a cuspidal edge if and only if $c_{36} \neq 0, c_{1}\left(c_{5}-s c_{36}\right)-\left(c_{3}^{\prime}+s c_{5}^{\prime}-s^{2} c_{36}^{\prime} / 2\right) \neq 0$.

(3) $F$ at $(s, t)$ is a swallowtail if and only if $c_{36} \neq 0, c_{5}-s c_{36} \neq 0, c_{1}\left(c_{5}-s c_{36}\right)-\left(c_{3}^{\prime}+s c_{5}^{\prime}-\right.$ $\left.s^{2} c_{36}^{\prime} / 2\right)=0$ and $-c_{1}^{2} c_{36}-c_{1}\left(c_{5}^{\prime}-s c_{36}^{\prime}\right)-\left(c_{1}^{\prime}\left(c_{5}-s c_{36}\right)+c_{1}\left(c_{5}^{\prime}-s c_{36}^{\prime}\right)-\left(c_{3}^{\prime \prime}+s c_{5}^{\prime \prime}-s^{2} c_{36}^{\prime \prime} / 2\right)\right) \neq 0$.

(4) $F$ at $(s, t)$ is a cuspidal lips if and only if $c_{36} \neq 0, c_{5}-s c_{36}=c_{3}^{\prime}+s c_{5}^{\prime}-s^{2} c_{36}^{\prime} / 2=0$ and $\operatorname{detHess} \lambda^{T}>0$.

(5) $F$ at $(s, t)$ is a cuspidal beaks if and only if $c_{36} \neq 0, c_{5}-s c_{36}=c_{3}^{\prime}+s c_{5}^{\prime}-s^{2} c_{36}^{\prime} / 2=0$, $\operatorname{det} \operatorname{Hess} \lambda^{T}<0$ and $-c_{1}^{2} c_{36}-c_{1}\left(c_{5}^{\prime}-s c_{36}^{\prime}\right)-c_{1}\left(c_{5}^{\prime}-s c_{36}^{\prime}\right)+\left(c_{3}^{\prime \prime}+s c_{5}^{\prime \prime}-s^{2} c_{36}^{\prime \prime} / 2\right) \neq 0$.

(6) $F$ at $(s, t)$ is a cuspidal cross cap if and only if $c_{36}=0, c_{36}^{\prime} \neq 0$ and $c_{1}\left(c_{5}-s c_{36}\right)-\left(c_{3}^{\prime}+\right.$ $\left.s c_{5}^{\prime}-s^{2} c_{36}^{\prime} / 2\right) \neq 0$.

Here, a map germ $f:\left(\mathbb{R}^{2}, \boldsymbol{a}\right) \longrightarrow\left(\mathbb{R}^{3}, \boldsymbol{b}\right)$ is called a cuspidal edge (respectively, swallowtail) if it is $\mathcal{A}$-equivalent to the germ $\left(u_{1}, u_{2}^{2}, u_{2}^{3}\right)$ (respectively, $\left(3 u_{1}^{4}+u_{1}^{2} u_{2}, 4 u_{1}^{3}+2 u_{1} u_{2}\right)$ ) (cf., Figure 2). Moreover, a map germ $f:\left(\mathbb{R}^{2}, \boldsymbol{a}\right) \longrightarrow\left(\mathbb{R}^{3}, \boldsymbol{b}\right)$ is called a cuspidal beaks (respectively, cuspidal cross cap) if it is $\mathcal{A}$-equivalent to the germ $\left(u_{2},-2 u_{1}^{3}+u_{2}^{2} u_{1}, 3 u_{1}^{4}-u_{1}^{2} u_{2}^{2}\right)$ (respectively, $\left.\left(u_{1}, u_{1} u_{2}^{3}, u_{2}^{2}\right)\right)$ (cf., Figure 3 ). 


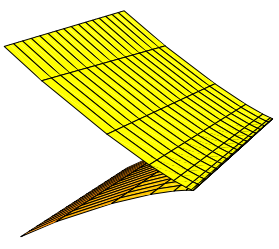

cuspital edge



cuspital beaks

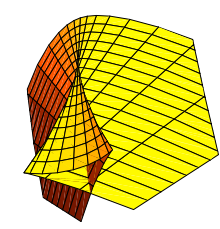

swallowtail

Figure 2

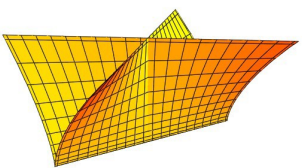

cuspidal cross cap

Figure 3

Proof. We see $\lambda^{T}=c_{3}(t)+s c_{5}(t)-s^{2} c_{36}(t) / 2$ and $\eta^{T}=c_{1}(\partial / \partial s)-\partial / \partial t$. The normal vector is $\boldsymbol{g}=\boldsymbol{a}_{1}+\boldsymbol{a}_{3}$. By definition, $F$ is front if and only if $\eta^{T} \boldsymbol{g} \neq 0$. Hence $\eta^{T} \boldsymbol{g}=-\boldsymbol{g}^{\prime}=c_{36} \boldsymbol{a}_{4}$. Thus we have (1).

The criterion for cuspidal edge is the following: $F$ at $(s, t)$ is a cuspidal edge if and only if $F$ is a front and $\eta^{T} \lambda^{T} \neq 0$. Hence $\eta^{T} \lambda^{T}=c_{1}\left(c_{5}-s c_{36}\right)-\left(c_{3}^{\prime}+s c_{5}^{\prime}-s^{2} c_{36}^{\prime} / 2\right)$, we have (2).

The criterion for swallowtail is the following: $F$ at $p$ is a swallowtail if and only if $F$ is a front, $d \lambda^{T} \neq 0, \eta^{T} \lambda^{T}=0$ and $\eta^{T} \eta^{T} \lambda^{T} \neq 0$. Applying this crieria, we have (3).

The criteria for cuspidal lips/beaks are the following: $F$ at $p$ is a cuspidal lips/beaks if and only if $F$ is a front, $d \lambda^{T}=0$ and $\operatorname{det} \operatorname{Hess} \lambda^{T}>0$ (lips). (for the beaks, $d \lambda^{T}=0$, $\operatorname{det} \operatorname{Hess} \lambda^{T}<0$ and $\left.\eta^{T} \eta^{T} \lambda \neq 0\right)$. Applying this crieria, we have (4) and (5).

If $c_{5} \neq 0$, we can parametrize $S(F)$ by $\gamma(t)=(s(t), t)$. Put

$$
\phi(t)=\operatorname{det}\left(F(\gamma), F(\gamma)^{\prime}, g(\gamma), g(\gamma)^{\prime}\right)(t) .
$$

Then $\phi(t)=\left(c_{1}(t)+s(t)^{\prime}\right) c_{36}(t)$. The criteria for cuspidal cross cap is the following: $F$ at $(s, t)$ is a cuspidal cross cap if and only if $F$ is not a front, $\eta^{T} \lambda^{T} \neq 0, \phi=0$ and $\phi^{\prime} \neq 0$. Applying this criteria, we have (6).

We also give the classification of singularities of the flat tangent TAdS-horocyclic surfaces. In this case, we have $c_{2}(t)=c_{3}(t)=c_{14}=0$ and all TAdS-horocycles are tangent to $\boldsymbol{a}_{1}$. We have the following corollary:

Corolary 5.2 Let $F$ be a flat tangent TAdS-horocyclic surface. Suppose $(s, t) \in S(F)$, where $s=0$ or $s=2 c_{5}(t) / c_{6}(t)$. Then the following assertions hold.

(1) $F$ at $(s, t)$ is a front if and only if $c_{6} \neq 0$.

(2) (a) $F$ at $\left(s=2 c_{5} / c_{6}, t\right)$ is a cuspidal edge if and only if $c_{5} c_{6}\left(c_{1}+s^{\prime}\right) \neq 0$.

(b) $F$ at $(0, t)$ is a cuspidal edge if and only if $c_{1} c_{5} c_{6} \neq 0$.

(3) (a) $F$ at $\left(s=2 c_{5} / c_{6}, t\right)$ is a swallowtail if and only if $c_{5} c_{6} \neq 0, c_{1}+s^{\prime}=0$ and $c_{1}\left(c_{6} s^{\prime}+\right.$ $\left.s c_{6}^{\prime}\right)+c_{5}\left(c_{1}^{\prime}+s^{\prime \prime}\right)-2 c_{1} c_{5}^{\prime} \neq 0$.

(b) $F$ at $(0, t)$ is a swallowtail if and only if $c_{5} c_{6} \neq 0, c_{1}=0$ and $c_{1}^{\prime} \neq 0$.

(4) $F$ at $(0, t)$ never be a cuspidal lips. 
(5) $F$ at $(0, t)$ is a cuspidal beaks if and only if $c_{6} \neq 0, c_{5}=0, c_{5}^{\prime} \neq 0$ and $c_{1}\left(c_{1} c_{6}+2 c_{5}^{\prime}\right) \neq 0$.

(6) $F$ at $(0, t)$ is a cuspidal cross cap if and only if $c_{6}=0, c_{6}^{\prime} \neq 0$ and $c_{1} c_{5} \neq 0$.

Proof. Substituting $c_{3} \equiv 0$ and $s=0$ in the formulas in Theorem 5.1.

We now consider the generic singularities of flat TAdS-horocyclic surfaces. As a result, we have the following theorem.

Theorem 5.3 (1) There exists a residual subset $\mathcal{O}_{1}^{T} \subset C^{\infty}\left(I, \mathfrak{f} \mathfrak{a} \mathfrak{h}^{T}(2,2)\right)$ such that for any $C \in \mathcal{O}_{1}^{T}$, singularities of $F_{\left(a_{1}, a_{2}, a_{3}\right)}^{T}$ are only cuspidal edge, swallowtail, cuspidal lips, cuspidal beaks or cuspidal cross cap.

(2) There exists a residual subset $\mathcal{O}_{2}^{T} \subset C^{\infty}\left(I, \mathfrak{f} \mathfrak{a} \mathfrak{h}_{t}^{T}(2,2)\right)$ such that for any $C \in \mathcal{O}_{2}^{T}$, singularities of $F_{\left(a_{1}, a_{2}, a_{3}\right)}^{T}$ are only cuspidal edge, swallowtail, cuspidal beaks or cuspidal cross cap.

Proof. (1) We consider the 2-jet space

$$
J^{2}\left(I, \mathfrak{f a h} \mathfrak{h}^{T}(2,2)\right) \cong I \times \mathbb{R}^{4} \times \mathbb{R}^{4} \times \mathbb{R}^{4}=\left\{(t, c, d, e) \mid t \in I, c, d, e \in \mathbb{R}^{4}\right\},
$$

where $c=\left(c_{1}, c_{3}, c_{5}, c_{6}\right), d=\left(d_{1}, d_{3}, d_{5}, d_{6}\right), e=\left(e_{1}, e_{3}, e_{5}, e_{6}\right)$. Define

$$
\begin{gathered}
S_{1}=\left\{c_{5}=0\right\}, S_{2}=\left\{c_{36}=0\right\}, S_{3}=\left\{c_{1}+s^{\prime}=0\right\}, S_{4}=\left\{d_{36}=0\right\}, \\
S_{5}=\left\{c_{5}-s c_{36}=0\right\}, S_{6}=\left\{d_{3}+s d_{5}-\frac{s^{2}}{2} d_{36}=0\right\} \\
S_{7}=\left\{c_{1}\left(c_{5}-s c_{36}\right)-\left(d_{3}+s d_{5}-\frac{s^{2}}{2} d_{36}\right)=0\right\} \\
S_{8}=\left\{-c_{1}^{2} c_{6}-c_{1}\left(d_{5}-s d_{36}\right)-\left(d_{1}\left(c_{5}-s c_{36}\right)+c_{1}\left(d_{5}-s d_{36}\right)-\left(s e_{5}-s^{2} e_{36} / 2\right)\right)=0\right\} .
\end{gathered}
$$

Here, $d_{36}=d_{3}+d_{6}$ and $e_{36}=e_{3}+e_{6}$. We see that $S_{1}$ is codimension one submanifolds and $S_{i}(i=2,3,4,5,6,7)$ is algebraic subset of codimension one. By the Thom's jet transversality theorem,

$\mathcal{O}_{1}^{T}=\left\{C \in C^{\infty}\left(I, \mathfrak{f a} \mathfrak{h}^{T}(2,2)\right) \mid j^{1} C\right.$ is transverse to

$$
\left.S_{2}, S_{2} \cap S_{5} \cap S_{6}, S_{2} \cap\left(S_{1} \cup S_{3} \cup S_{7}\right), S_{5} \cap S_{6} \cap\left(S_{2} \cup S_{8}\right), S_{5} \cap S_{6} \cap S_{2}, S_{7} \cap\left(S_{2} \cup S_{5} \cup S_{8}\right)\right\}
$$

is an open and dense in $C^{\infty}\left(I, \mathfrak{f} \mathfrak{a} \mathfrak{h}^{T}(2,2)\right)$. On the other hand, one can easily see that Theorem 5.1 implies that $\mathcal{O}_{1}^{T}$ satisfies the required condition.

(2) We now consider the 2-jet space

$$
\left.J^{2}(I, \mathfrak{f a h})_{t}^{T}(2,2)\right) \cong I \times \mathbb{R}^{3} \times \mathbb{R}^{3} \times \mathbb{R}^{3}=\left\{(t, c, d, e) \mid t \in I, c, d, e \in \mathbb{R}^{3}\right\},
$$

where $c=\left(c_{1}, c_{5}, c_{6}\right), d=\left(d_{1}, d_{5}, d_{6}\right), e=\left(e_{1}, e_{5}, e_{6}\right)$. We also define

$$
\begin{gathered}
S_{1}=\left\{c_{5}=0\right\}, S_{2}=\left\{c_{6}=0\right\}, S_{3}=\left\{c_{1}=0\right\}, \\
S_{4}=\left\{c_{5}-s c_{6}=0\right\}, S_{5}=\left\{c_{1}\left(c_{5}-s c_{6}\right)-\left(s d_{5}-\frac{s^{2}}{2} d_{6}\right)=0\right\}, \\
S_{6}=\left\{-c_{1}^{2} c_{6}-c_{1}\left(d_{5}-s d_{6}\right)-\left(d_{1}\left(c_{5}-s c_{6}\right)+c_{1}\left(d_{5}-s d_{6}\right)-\left(s e_{5}-s^{2} e_{6} / 2\right)\right)\right\}, \\
S_{7}=\left\{d_{6}=0\right\}, S_{8}=\left\{d_{1}=0\right\}, S_{9}=\left\{d_{5}=0\right\}, S_{10}=\left\{c_{1}\left(c_{1} c_{6}+2 d_{5}\right)\right\} .
\end{gathered}
$$


We have the following open dense subset of $C^{\infty}\left(I, \mathfrak{f} \mathfrak{a h} \mathfrak{h}_{t}^{T}(2,2)\right)$ :

$\mathcal{O}_{2}^{T}=\left\{C \in C^{\infty}(I, \mathfrak{f} \mathfrak{a h})_{t}^{T}(2,2)\right) \mid j^{1} C$ is transverse to

$$
\left.S_{2}, S_{2} \cap\left(S_{1} \cup S_{3} \cup S_{7}\right), S_{1} \cap\left(S_{2} \cup S_{9} \cup S_{10}\right), S_{3} \cap\left(S_{1} \cup S_{2} \cup S_{8}\right), S_{5} \cap\left(S_{2} \cup S_{4} \cup S_{6}\right)\right\} .
$$

By Corollary 5.2, $\mathcal{O}_{2}^{T}$ satisfies the required condition.

\subsection{Singularities of flat SAdS-horocyclic surfaces}

In this section, we will give the classifications of singularities of flat SAdS-horocyclic surfaces by the same arguments as those of the flat TAdS-horocyclic surfaces. We only state the fundamental data here and omit the detailed arguments. By straightforward calculation, the singular points $(s, t)$ of $F_{\left(a_{1}, a_{3}, a_{4}\right)}^{S}$ satisfy that

$$
\Theta^{S}(s, t)=c_{14}(t) s^{2}+2 c_{5}(t) s+2 c_{1}(t)=0
$$

So that the flat SAdS-horocyclic surfaces $F_{\left(a_{1}, a_{3}, a_{4}\right)}^{S}$ has singular points $(s, t)$ if and only if $\Theta^{S}(s, t)=0$ has real roots. Under the condition that $c_{14} \neq 0$, this condition is equivalent to the following condition

$$
\Delta^{S}(t)=c_{5}^{2}(t)-2 c_{1}(t) c_{14}(t) \geq 0 .
$$

We say that $F_{\left(a_{1}, a_{3}, a_{4}\right)}^{S}$ is a flat tangent AdS-horocyclic surface if $c_{1}(t)=c_{2}(t)=c_{36}=0$. We also define a linear subspaces of $\mathfrak{s o}(2,2)$ by

$$
\mathfrak{f a h}_{t}^{S}(2,2)=\left\{C \in \mathfrak{s o}(2,2) \mid c_{1}(t)=c_{2}(t)=c_{36}=0\right\} .
$$

We call space $C^{\infty}\left(I, \mathfrak{f a h}_{t}^{S}(2,2)\right)$ equipped with Whitney $C^{\infty}$-topology the space of flat tangent SAdS-horocyclic surfaces. In this space, one of the branches of the singularities of the flat SAdS-horocyclic surface is always located on the image of $\boldsymbol{a}_{1}$.

We now give the classification of singularities of the flat SAdS-horocyclic surface. In this case, we see that the normal vector of $F_{\left(a_{1}, a_{3}, a_{4}\right)}^{S}$ is well-defined and it is $\boldsymbol{g}=\boldsymbol{a}_{1}+\boldsymbol{a}_{3}$. This means that $F_{\left(a_{1}, a_{3}, a_{4}\right)}^{S}$ is a frontal. Moreover we see that

$$
\begin{gathered}
S\left(F_{\left(a_{1}, a_{3}, a_{4}\right)}^{S}\right)=\left\{(s, t) \mid \widehat{c}_{1}(t)=0\right\} \\
\eta^{S}(s, t)=\operatorname{ker} d F_{\left(a_{1}, a_{3}, a_{4}\right)}^{S}(s ; t)=c_{3}(t) \frac{\partial}{\partial s}-\frac{\partial}{\partial t}, \\
\lambda^{S}(s, t)=c_{1}(t)+s c_{5}(t)+\frac{s^{2} c_{14}(t)}{2} .
\end{gathered}
$$

For $F=F_{\left(a_{1}, a_{3}, a_{4}\right)}^{S}$, by almost the same proofs as those of Theorem 5.1 and Corollary 5.2, we have the following.

Theorem 5.4 Let $F$ be a flat $S A d S$-horocyclic surface and $(s, t) \in S(F)$. Then the following assertions hold.

(1) $F$ at $(s, t)$ is a front if and only if $c_{14} \neq 0$.

(2) $F$ at $(s, t)$ is a cuspidal edge if and only if $c_{14} \neq 0, c_{3}\left(c_{5}+s c_{14}\right)-\left(c_{1}^{\prime}+s c_{5}^{\prime}+s^{2} c_{14}^{\prime} / 2\right) \neq 0$.

(3) $F$ at $(s, t)$ is a swallowtail if and only if $c_{14} \neq 0, c_{5}+s c_{14} \neq 0, c_{3}\left(c_{5}+s c_{14}\right)-\left(c_{1}^{\prime}+s c_{5}^{\prime}+\right.$ $\left.s^{2} c_{14}^{\prime} / 2\right)=0$ and $c_{3}^{2} c_{14}-c_{3}\left(c_{5}^{\prime}+s c_{14}^{\prime}\right)-\left(c_{3}^{\prime}\left(c_{5}+s c_{14}\right)-c_{3}\left(c_{5}^{\prime}+s c_{14}^{\prime}\right)-\left(c_{1}^{\prime \prime}+s c_{5}^{\prime \prime}+s^{2} c_{14}^{\prime \prime} / 2\right)\right) \neq 0$.

(4) $F$ at $(s, t)$ is a cuspidal lips if and only if $c_{14} \neq 0, c_{5}+s c_{14}=c_{1}^{\prime}+s c_{5}^{\prime}+s^{2} c_{14}^{\prime} / 2=0$ and $\operatorname{det} \operatorname{Hess} \lambda^{S}>0$. 
(5) $F$ at $(s, t)$ is a cuspidal beaks if and only if $c_{14} \neq 0, c_{5}+s c_{14}=c_{1}^{\prime}+s c_{5}^{\prime}+s^{2} c_{14}^{\prime} / 2=0$, $\operatorname{det} \operatorname{Hess} \lambda^{S}<0$ and $c_{3}^{2} c_{14}-c_{3}\left(c_{5}^{\prime}+s c_{14}^{\prime}\right)-\left(c_{3}^{\prime}\left(c_{5}+s c_{14}\right)-c_{3}\left(c_{5}^{\prime}+s c_{14}^{\prime}\right)-\left(c_{1}^{\prime \prime}+s c_{5}^{\prime \prime}+s^{2} c_{14}^{\prime \prime} / 2\right)\right) \neq 0$. (6) $F$ at $(s, t)$ is a cuspidal cross cap if and only if $c_{14}=0, c_{14}^{\prime} \neq 0$ and $c_{3}\left(c_{5}+s c_{14}\right)-\left(c_{1}^{\prime}+\right.$ $\left.s c_{5}^{\prime}+s^{2} c_{14}^{\prime} / 2\right) \neq 0$.

Corolary 5.5 Let $F$ be a flat tangent $S A d S$-horocyclic surface. Suppose $(s, t) \in S(F)$, where $s=0$ or $s=-2 c_{5}(t) / c_{4}(t)$. Then the following assertions hold.

(1) $F$ at $(s, t)$ is a front if and only if $c_{4} \neq 0$.

(2) (a) $F$ at $\left(s=-2 c_{5} / c_{4}, t\right)$ is a cuspidal edge if and only if $c_{4} c_{5}\left(c_{3}+s^{\prime}\right) \neq 0$.

(b) $F$ at $(0, t)$ is a cuspidal edge if and only if $c_{3} c_{4} c_{5} \neq 0$.

(3) (a) $F$ at $\left(s=-2 c_{5} / c_{4}, t\right)$ is a swallowtail if and only if $c_{4} c_{5} \neq 0, c_{3}+s^{\prime}=0$ and $c_{3}\left(s c_{4}^{\prime}-\right.$ $\left.c_{4} s^{\prime}\right)+c_{5}\left(c_{3}^{\prime}+s^{\prime \prime}\right) \neq 0$.

(b) $F$ at $(0, t)$ is a swallowtail if and only if $c_{4} c_{5} \neq 0, c_{3}=0$ and $c_{3}^{\prime} \neq 0$.

(4) F at $(0, t)$ never be a cuspidal lips.

(5) $F$ at $(0, t)$ is a cuspidal beaks if and only if $c_{4} \neq 0, c_{5}=0, c_{5}^{\prime} \neq 0$ and $c_{3}\left(c_{3} c_{4}-2 c_{5}^{\prime}\right) \neq 0$.

(6) $F$ at $(0, t)$ is a cuspidal cross cap if and only if $c_{4}=0, c_{4}^{\prime} \neq 0$ and $c_{3} c_{5} \neq 0$.

We can also study the generic singularities of flat SAdS-horocyclic surfaces by the same arguments as those of Theorem 5.3. We only give the result.

Theorem 5.6 (1) There exists a residual subset $\mathcal{O}_{1}^{S} \subset C^{\infty}\left(I, \mathfrak{f a h}^{S}(2,2)\right)$ such that for any $C \in \mathcal{O}_{1}^{S}$, singularities of $F_{\left(a_{1}, a_{3}, a_{4}\right)}^{S}$ are only cuspidal edge, swallowtail, cuspidal lips, cuspidal beaks or cuspidal cross cap.

(2) There exists a residual subset $\mathcal{O}_{2}^{S} \subset C^{\infty}\left(I, \mathfrak{f a h}_{t}^{S}(2,2)\right)$ such that for any $C \in \mathcal{O}_{2}^{S}$, singularities of $F_{\left(a_{1}, a_{3}, a_{4}\right)}^{S}$ are only cuspidal edge, swallowtail, cuspidal beaks or cuspidal cross cap.

\section{Duality between $H_{1}^{3}$ and $\Lambda^{3}$}

In this section we consider the duality of the singularities between curves and surfaces in $H_{1}^{3}$ and $\Lambda^{3}$ by using the Legendrian dualities developed in [3]. We first review the $\Delta_{2}$-duality.

(a) $H_{1}^{3} \times \Lambda^{3} \supset \Delta_{2}=\{(\boldsymbol{v}, \boldsymbol{w}) \mid\langle\boldsymbol{v}, \boldsymbol{w}\rangle=-1\}$,

(b) $\pi_{21}: \Delta_{2} \longrightarrow H_{1}^{3}, \pi_{22}: \Delta_{2} \longrightarrow \Lambda^{3}$,

(c) $\theta_{21}=\langle d \boldsymbol{v}, \boldsymbol{w}\rangle\left|\Delta_{2}, \theta_{22}=\langle\boldsymbol{v}, d \boldsymbol{w}\rangle\right| \Delta_{2}$.

Where, $\pi_{21}(\boldsymbol{v}, \boldsymbol{w})=\boldsymbol{v}, \pi_{22}(\boldsymbol{v}, \boldsymbol{w})=\boldsymbol{w},\langle d \boldsymbol{v}, \boldsymbol{w}\rangle=-w_{1} d v_{1}-w_{2} d v_{2}+w_{3} d v_{3}+w_{4} d v_{4}$ and $\langle\boldsymbol{v}, d \boldsymbol{w}\rangle=-v_{1} d w_{1}-v_{2} d w_{2}+v_{3} d w_{3}+v_{4} d w_{4}$ are one-forms on $\mathbb{R}_{2}^{4} \times \mathbb{R}_{2}^{4}$. We remark that $\theta_{21}^{-1}(0)$ and $\theta_{22}^{-1}(0)$ define the same tangent hyperplane field over $\Delta_{2}$ which is denoted by $K_{2}$. We have shown in [3] that $\left(\Delta_{2}, K_{2}\right)$ is a contact manifold such that both of the fibrations $\pi_{21}$ and $\pi_{22}$ are Legendrian fibration. In [3] we defined four Legendrian fibrations $\left(\Delta_{i}, K_{i}\right)(i=1,2,3,4)$ such that these are contact equivalent to each other. Here, we only use $\left(\Delta_{2}, K_{2}\right)$. For definitions and basic results of Legendrian fibrations, see $[1,3]$. We say that smooth mappings $f: U \longrightarrow H_{1}^{3}$ and $g: U \longrightarrow \Lambda^{3}$ are $\Delta_{2}$-dual if there exist a mapping $\mathcal{L}_{(f, g)}: U \longrightarrow \Delta_{2}$ such that $\pi_{21} \circ \mathcal{L}_{(f, g)}=f$, $\pi_{22} \circ \mathcal{L}_{(f, g)}=g$ and $\mathcal{L}_{(f, g)}^{*} \theta_{21}=0$. If $\boldsymbol{X}: U \longrightarrow H_{1}^{3}$ is a timelike immersion, the AdS-nullcone Gauss image $\mathbb{G}_{n}^{ \pm}$of $\boldsymbol{X}$ and $\boldsymbol{X}$ are $\Delta_{2}$-dual.

We now define two surfaces by using the pseudo-orthonormal frame $\left\{\boldsymbol{a}_{1}, \boldsymbol{a}_{2}, \boldsymbol{a}_{3}, \boldsymbol{a}_{4}\right\}$ defined 
in $\S 4$ as follow:

$$
G^{T}:[0,2 \pi) \times I \longrightarrow \Lambda^{3}, \quad G^{T}(\theta, t)=\boldsymbol{a}_{1}(t)+\cos \theta \boldsymbol{a}_{3}(t)+\sin \theta \boldsymbol{a}_{4}(t)
$$

and

$$
G^{S}: \mathbb{R} \times I \longrightarrow \Lambda^{3}, \quad G^{S}(\theta, t)=\boldsymbol{a}_{1}(t)+\sinh \theta \boldsymbol{a}_{2}(t)+\cosh \theta \boldsymbol{a}_{3}(t) .
$$

We call them a nullcone circular surface and a nullcone hyperboloid respectively. For any fixed $t_{0} \in I$, we have a circle $G^{T}\left(\theta, t_{0}\right)=\boldsymbol{a}_{1}\left(t_{0}\right)+\cos \theta \boldsymbol{a}_{3}\left(t_{0}\right)+\sin \theta \boldsymbol{a}_{4}\left(t_{0}\right)$ and a hyperbola $G^{S}\left(\theta, t_{0}\right)=\boldsymbol{a}_{1}\left(t_{0}\right)+\sinh \theta \boldsymbol{a}_{2}\left(t_{0}\right)+\cosh \theta \boldsymbol{a}_{3}\left(t_{0}\right)$ respectively. We remark that both of the above curves pass through the point $\boldsymbol{g}\left(t_{0}\right)=\boldsymbol{a}_{1}\left(t_{0}\right)+\boldsymbol{a}_{3}\left(t_{0}\right)$ and we call them a generating circle and a generating hyperbola respectively.

\subsection{Singularities of AdS-flat tangent nullcone circular surface}

In this subsection, we give a classification of singularities of a special kind of nullcone circular surfaces. By a straightforward calculation, we have

$$
\begin{aligned}
& \frac{\partial G^{T}}{\partial \theta}=-\sin \theta \boldsymbol{a}_{3}(t)+\cos \theta \boldsymbol{a}_{4}(t), \\
& \frac{\partial G^{T}}{\partial t}=\boldsymbol{a}_{1}^{\prime}(t)+\cos \theta \boldsymbol{a}_{3}^{\prime}(t)+\sin \theta \boldsymbol{a}_{4}^{\prime}(t) .
\end{aligned}
$$

Thus, $\boldsymbol{a}_{1}(t)$ is an Anti de Sitter normal (briefly, AdS-normal) at any regular point $(\theta, t)$ if and only if

$$
\left\langle\frac{\partial G^{T}}{\partial t}(\theta, t), \boldsymbol{a}_{1}(t)\right\rangle=-\cos \theta c_{2}(t)-\sin \theta c_{3}(t)=0
$$

for any $\theta \in[0,2 \pi]$. This condition is equivalent to the following

$$
c_{2}(t)=c_{3}(t)=0 .
$$

In this case, we have

$$
\frac{\partial G^{T}}{\partial t}=\left(c_{1}(t)+c_{4}(t) \cos \theta+c_{5} \sin \theta\right) \boldsymbol{a}_{2}(t)-c_{6}(t) \sin \theta \boldsymbol{a}_{3}(t)+c_{6}(t) \cos \theta \boldsymbol{a}_{4}(t) .
$$

It follows that

$$
S\left(G^{T}\right)=\left\{(\theta, t) \mid c_{1}(t)+c_{4}(t) \cos \theta+c_{5} \sin \theta=0\right\} .
$$

So that $(0, t)$ is always singular if and only if $c_{14}(t)=0$. In this case, $\boldsymbol{g}^{\prime}(t)=c_{6}(t) \boldsymbol{a}_{4}(t)$ and the generating circle is tangent to $\boldsymbol{g}(t)$. We call $G^{T}$ an $A d S$-flat tangent nullcone circular surface if $c_{2}(t)=c_{3}(t)=c_{14}(t)=0$. This condition is equivalent to the condition that $F_{\left(a_{1}, a_{2}, a_{3}\right)}^{T}$ is a flat tangent TAdS-horocyclic surface such that one of the branches of singularities is located on the set $(0, t)$. In this case, we see that the normal vector of $G^{T}$ is well-defined and it is $\boldsymbol{a}_{1}$. This means that $G^{T}$ is a frontal. Moreover we see that

$$
\begin{gathered}
\zeta^{T}(s, t)=\operatorname{ker} d G^{T}(s, t)=c_{6}(t) \frac{\partial}{\partial \theta}-\frac{\partial}{\partial t}, \\
\xi^{T}(s, t)=-c_{1}(t)-\cos \theta c_{4}(t)-\sin \theta c_{5}(t) .
\end{gathered}
$$

We call $\xi^{T}$ the T-signed null area density function. By using the same method as the proof of Theorem 5.1, we can show the following theorem.

Theorem 6.1 Let $G^{T}$ be an AdS-flat tangent nullcone circular surface. Suppose $(\theta, t) \in$ $S\left(G^{T}\right)$, where $\theta=0$ or $\theta$ satisfies the equation $c_{1}(t)(1-\cos \theta)+c_{5} \sin \theta=0$. Then the following assertions hold. 
(1) $G^{T}$ at $(\theta, t)$ is a front if and only if $c_{1} \neq 0$.

(2) (a) $G^{T}$ at $(\theta, t)$ is a cuspidal edge if and only if $c_{1} \neq 0, c_{T}:=\left(c_{4}^{\prime}-c_{5} c_{6}\right) \cos \theta+\left(c_{5}^{\prime}+\right.$ $\left.c_{4} c_{6}\right) \sin \theta+c_{1}^{\prime} \neq 0$.

(b) $G^{T}$ at $(0, t)$ is a cuspidal edge if and only if $c_{1} c_{5} c_{6} \neq 0$.

(3) (a) $G^{T}$ at $(\theta, t)$ is a swallowtail if and only if $c_{1} \neq 0, c_{4} \sin \theta-c_{5} \cos \theta \neq 0, c_{T}=0$ and $c_{6}\left[\left(c_{5}^{\prime}+c_{4} c_{6}\right) \cos \theta-\left(c_{4}^{\prime}-c_{5} c_{6}\right) \sin \theta\right]-c_{T}^{\prime} \neq 0$.

(b) $G^{T}$ at $(0, t)$ is a swallowtail if and only if $c_{1} c_{5} \neq 0, c_{6}=0$ and $c_{6}^{\prime} \neq 0$.

(4) $G^{T}$ at $(0, t)$ never be a cuspidal lips.

(5) $G^{T}$ at $(0, t)$ is a cuspidal beaks if and only if $c_{1} \neq 0, c_{5}=0, c_{5}^{\prime} \neq 0$ and $c_{6}\left(c_{4} c_{6}+2 c_{5}^{\prime}\right) \neq 0$.

(6) $G^{T}$ at $(0, t)$ is a cuspidal cross cap if and only if $c_{5} c_{6} \neq 0, c_{1}=0$ and $c_{1}^{\prime} \neq 0$.

For the proof of above theorem, we can use almost the same way as those of Theorem 5.1. We will omit the detailed arguments and only state the fundamental data here.

$$
\begin{aligned}
d \xi^{T} & =-\left(c_{1}^{\prime}+c_{4}^{\prime} \cos \theta+c_{5}^{\prime} \sin \theta\right) d t+\left(c_{4} \sin \theta-c_{5} \cos \theta\right) d \theta, \\
\zeta^{T} \xi^{T} & =\left(c_{4}^{\prime}-c_{5} c_{6}\right) \cos \theta+\left(c_{5}^{\prime}+c_{4} c_{6}\right) \sin \theta+c_{1}^{\prime}=: c_{T}, \\
\zeta^{T} \zeta^{T} \xi^{T} & =c_{6}\left[\left(c_{5}^{\prime}+c_{4} c_{6}\right) \cos \theta-\left(c_{4}^{\prime}-c_{5} c_{6}\right) \sin \theta\right]-c_{T}^{\prime}, \\
\operatorname{det} \operatorname{Hess} \xi^{T} & =-\left(c_{4} \cos \theta+c_{5} \sin \theta\right)\left(c_{1}^{\prime \prime}+c_{4}^{\prime \prime} \cos \theta+c_{5}^{\prime \prime} \sin \theta\right)-\left(c_{4}^{\prime} \sin \theta-c_{5}^{\prime} \cos \theta\right)^{2} .
\end{aligned}
$$

Moreover, if $c_{5} \neq 0$, we can parametrize $S\left(G^{T}\right)$ by $\gamma(t)=(\theta(t), t)$. Put

$$
\phi(t)=\operatorname{det}\left(G^{T}(\gamma), G^{T}(\gamma)^{\prime}, \boldsymbol{a}_{1}(\gamma), \boldsymbol{a}_{1}(\gamma)^{\prime}\right)(t) .
$$

Then $\phi(t)=c_{1}(t)\left(c_{6}(t)+\theta^{\prime}(t)\right)$. We remark that we can also use the admissible lift which was developed in [6] to instead of $\phi(t)$. Since the key of the criterion for cuspidal cross cap is to find some non-degenerate normal. As it turns out, $\boldsymbol{a}_{1}$ is just one of the candidate what we wanted, so that we use $\phi(t)$ here.

On the other hand, we can show that $\left\langle F_{\left(a_{1}, a_{2}, a_{3}\right)}^{T}(s, t), \boldsymbol{g}(t)\right\rangle=\left\langle\boldsymbol{a}_{1}(t), G^{T}(\theta, t)\right\rangle=-1$. Therefore, we have two well defined mappings

$$
\begin{aligned}
& \mathcal{L}_{\left(F_{\left(a_{1}, a_{2}, a_{3}\right)}^{T}, g\right)}: J \times I \longrightarrow \Delta_{2}, \\
& \mathcal{L}_{\left(a_{1}, G^{T}\right)}:[0,2 \pi) \times I \longrightarrow \Delta_{2} .
\end{aligned}
$$

Since $\boldsymbol{g}(t)$ (respectively, $\boldsymbol{a}_{1}(t)$ ) is the normal of $F_{\left(a_{1}, a_{2}, a_{3}\right)}^{T}(s, t)$ (respectively, $G^{T}(\theta, t)$ ), it follows that $\mathcal{L}_{\left(F_{\left(a_{1}, a_{2}, a_{3}\right)}, g\right)}$ (respectively, $\left.\mathcal{L}_{\left(a_{0}, G^{T}\right)}\right)$ is an integrable mapping with respect to $K_{2}$. So that $F_{\left(a_{1}, a_{2}, a_{3}\right)}^{T}(s, t)$ and $\boldsymbol{g}(t)$ (respectively, $\boldsymbol{a}_{1}(t)$ and $G^{T}(\theta, t)$ ) are the $\Delta_{2}$-duality.

Comparing Theorem 6.1 with Corollary 5.2 when singular point is $(0, t)$, we observe a certain duality between the swallowtail and cuspidal cross cap as pointed out by many researchers, for example, $[6,19,20,26]$. We can also observe a self-duality of cuspidal edge. Furthermore, we can show the following result as a consequence of Theorem 6.1.

Corollary 6.2 Let $F_{\left(a_{1}, a_{2}, a_{3}\right)}^{T}$ be a flat tangent TAdS-horocyclic surface. Then in the case when the germ of the surface at $(0, t)$ is the cuspidal cross cap, the germ of $\widetilde{\boldsymbol{g}}: I \longrightarrow T_{1}^{2}$ at $t$ is the ordinary cusp.

Proof. Since $F_{\left(a_{1}, a_{2}, a_{3}\right)}^{T}$ is a flat tangent TAdS-horocyclic surface, it follows that $G^{T}$ is also an AdS-flat tangent nullcone circular surface. By the duality between the swallowtail and cuspidal cross cap, in the case when the germ of $F_{\left(a_{1}, a_{2}, a_{3}\right)}^{T}$ at $(0, t)$ is the cuspidal cross cap, the germ of $\widetilde{\boldsymbol{g}}$ at $(0, t)$ is the swallowtail. 
On the other hand, since $\pi_{22}: \Delta_{2} \longrightarrow \Lambda^{3}$ is a Legendrian fibration and the germ of $G^{T}$ has a Legendrian lift into $\Delta_{2}, G^{T}$ is a wavefront in $\Lambda^{3}$. According to the general Legendrian and Lagrangian singularity theory [1], $\widetilde{G^{T}}$ can be viewed as a Lagrangian map. By the relation between wavefront and caustics, the germ of $G^{T}$ is the swallowtail if and only if the caustics of $\widetilde{G^{T}}$ is the ordinary cusp. Moreover, the caustics of $\widetilde{G^{T}}$ is the image of $\widetilde{\boldsymbol{g}}$. So we complete the proof.

\subsection{Singularities of AdS-flat tangent nullcone hyperboloid}

In this section, we study the classification of singularities of a special kind of nullcone hyperboloid by using the similar way as those of nullcone circular surfaces. By straightforward calculation, we have

$$
\begin{aligned}
\frac{\partial G^{S}}{\partial \theta} & =\cosh \theta \boldsymbol{a}_{2}(t)+\sinh \theta \boldsymbol{a}_{3}(t), \\
\frac{\partial G^{S}}{\partial t} & =\boldsymbol{a}_{1}^{\prime}(t)+\sinh \theta \boldsymbol{a}_{2}^{\prime}(t)+\cosh \theta \boldsymbol{a}_{3}^{\prime}(t) .
\end{aligned}
$$

Thus, $\boldsymbol{a}_{1}(t)$ is an Anti de Sitter normal (briefly, AdS-normal) at any regular point $(\theta, t)$ if and only if

$$
\left\langle\frac{\partial G^{S}}{\partial t}(\theta, t), \boldsymbol{a}_{1}(t)\right\rangle=-\sinh \theta c_{1}(t)-\cosh \theta c_{2}(t)=0
$$

for any $\theta \in \mathbb{R}$. This condition is equivalent to the following

$$
c_{1}(t)=c_{2}(t)=0 \text {. }
$$

In this case, we have

$$
\frac{\partial G^{S}}{\partial t}=\cosh \theta \boldsymbol{a}_{2}(t)+\sinh \theta \boldsymbol{a}_{3}+\left(c_{3}(t)+c_{5} \sinh \theta+c_{6}(t) \cosh \theta\right) \boldsymbol{a}_{4}(t) .
$$

It follows that

$$
S\left(G^{S}\right)=\left\{(\theta, t) \mid c_{3}(t)+c_{5} \sinh \theta+c_{6}(t) \cosh \theta=0\right\} .
$$

So that $(0, t)$ is always singular if and only if $c_{36}(t)=0$. In this case, $\boldsymbol{g}^{\prime}(t)=c_{4}(t) \boldsymbol{a}_{2}(t)$ and the generating hyperbola is tangent to $\boldsymbol{g}(t)$. We call $G^{S}$ an AdS-flat tangent nullcone hyperboloid if $c_{1}(t)=c_{2}(t)=c_{36}(t)=0$. This condition is equivalent to the condition that $F_{\left(a_{1}, a_{3}, a_{4}\right)}^{S}$ is a flat tangent SAdS-horocyclic surface such that one of the branches of singularities is located on the set $(0, t)$. In this case, we see that the normal vector of $G^{S}$ is well-defined and it is $\boldsymbol{a}_{1}$. This means that $G^{S}$ is a frontal. Moreover we see that

$$
\begin{gathered}
\zeta^{S}(s, t)=\operatorname{ker} d G^{S}(s, t)=c_{4}(t) \frac{\partial}{\partial \theta}-\frac{\partial}{\partial t}, \\
\xi^{S}(s, t)=c_{3}(t)+\sinh \theta c_{5}(t)+\cosh \theta c_{6}(t) .
\end{gathered}
$$

We call $\xi^{S}$ the $S$-signed null area density function. By using the same method with Theorem 6.1 , we can show the following theorem.

Theorem 6.3 Let $G^{S}$ be an AdS-flat tangent nullcone hyperboloid. Suppose $(\theta, t) \in S\left(G^{S}\right)$, where $\theta=0$ or $\theta$ satisfies the equation $\left.c_{3}(t)(1-\cosh \theta)+\sinh \theta c_{5}(t)\right)=0$. Then the following assertions hold.

(1) $G^{S}$ at $(\theta, t)$ is a front if and only if $c_{3} \neq 0$.

(2) (a) $G^{S}$ at $(\theta, t)$ is a cuspidal edge if and only if $c_{3} \neq 0, c_{S}:=\left(c_{4} c_{5}-c_{6}^{\prime}\right) \cosh \theta+\left(c_{4} c_{6}-\right.$ $\left.c_{5}^{\prime}\right) \sinh \theta-c_{3}^{\prime} \neq 0$.

(b) $G^{S}$ at $(0, t)$ is a cuspidal edge if and only if $c_{3} c_{4} c_{5} \neq 0$. 
(3) (a) $G^{S}$ at $(\theta, t)$ is a swallowtail if and only if $c_{3} \neq 0, c_{5} \cosh \theta+c_{6} \sin \theta \neq 0, c_{S}=0$ and $c_{4}\left[\left(c_{4} c_{5}-c_{6}^{\prime}\right) \sinh \theta+\left(c_{4} c_{6}-c_{5}^{\prime}\right) \cosh \theta\right]-c_{S}{ }^{\prime} \neq 0$.

(b) $G^{S}$ at $(0, t)$ is a swallowtail if and only if $c_{3} c_{5} \neq 0, c_{4}=0$ and $c_{4}^{\prime} \neq 0$.

(4) $G^{S}$ at $(0, t)$ never be a cuspidal lips.

(5) $G^{S}$ at $(0, t)$ is a cuspidal beaks if and only if $c_{3} \neq 0, c_{5}=0, c_{5}^{\prime} \neq 0$ and $c_{4}\left(c_{4} c_{6}-2 c_{5}^{\prime}\right) \neq 0$.

(6) $G^{S}$ at $(0, t)$ is a cuspidal cross cap if and only if $c_{4} c_{5} \neq 0, c_{3}=0$ and $c_{3}^{\prime} \neq 0$.

Corollary 6.4 Let $F_{\left(a_{1}, a_{3}, a_{4}\right)}^{S}$ be a flat tangent SAdS-horocyclic surface. Then in the case when the germ of the surface at $(0, t)$ is the cuspidal cross cap, the germ of $\widetilde{\boldsymbol{g}}: I \longrightarrow T_{1}^{2}$ at $t$ is the ordinary cusp.

We now summarize the dual relations between singularity, when singular point is $(0, t)$, in the following table. We see that the self duality of cuspidal beaks does not hold where it holds on the flat great circular surfaces [20, Section 7].

Dualities conditions for singularity

\begin{tabular}{|c|c|c|c|c|c|c|}
\hline Surfaces & duality & $\mathrm{S}=\{(0, \mathrm{t})\}$ & $\mathrm{CE}$ & SW & $\overline{\mathrm{CCR}}$ & CBK \\
\hline$F_{\left(a_{1}, a_{2}, a_{3}\right)}^{T}$ & $\begin{array}{c}c_{2}=0 \\
c_{14}=0\end{array}$ & $c_{3}=0$ & $c_{1} c_{5} c_{6} \neq 0$ & $\begin{array}{c}c_{5} c_{6} \neq 0 \\
c_{1}=0, c_{1}^{\prime} \neq 0\end{array}$ & $\begin{array}{c}c_{1} c_{5} \neq 0 \\
c_{6}=0, c_{6}^{\prime} \neq 0\end{array}$ & $\begin{array}{c}c_{1} c_{6} \neq 0, \\
c_{5}=0, c_{5}^{\prime} \neq 0 \\
c_{1} c_{6}+2 c_{5}^{\prime} \neq 0\end{array}$ \\
\hline$G^{T}$ & $\begin{array}{l}c_{2}=0 \\
c_{3}=0\end{array}$ & $c_{14}=0$ & $c_{1} c_{5} c_{6} \neq 0$ & $\begin{array}{c}c_{1} c_{5} \neq 0 \\
c_{6}=0, c_{6}^{\prime} \neq 0\end{array}$ & $\begin{array}{c}c_{5} c_{6} \neq 0 \\
c_{1}=0, c_{1}^{\prime} \neq 0\end{array}$ & $\begin{array}{c}c_{1} c_{6} \neq 0, \\
c_{5}=0, c_{5}^{\prime} \neq 0 \\
c_{4} c_{6}+2 c_{5}^{\prime} \neq 0\end{array}$ \\
\hline$F_{\left(a_{1}, a_{3}, a_{4}\right)}^{S}$ & $\begin{array}{c}c_{2}=0 \\
c_{36}=0\end{array}$ & $c_{1}=0$ & $c_{3} c_{4} c_{5} \neq 0$ & $\begin{array}{c}c_{4} c_{5} \neq 0 \\
c_{3}=0, c_{3}^{\prime} \neq 0\end{array}$ & $\begin{array}{c}c_{3} c_{5} \neq 0 \\
c_{4}=0, c_{4}^{\prime} \neq 0\end{array}$ & $\begin{array}{c}c_{3} c_{4} \neq 0, \\
c_{5}=0, c_{5}^{\prime} \neq 0 \\
c_{3} c_{4}-2 c_{5}^{\prime} \neq 0\end{array}$ \\
\hline$G^{S}$ & $\begin{array}{l}c_{1}=0 \\
c_{2}=0\end{array}$ & $c_{36}=0$ & $c_{3} c_{4} c_{5} \neq 0$ & $\begin{array}{c}c_{3} c_{5} \neq 0 \\
c_{4}=0, c_{4}^{\prime} \neq 0\end{array}$ & $\begin{array}{c}c_{4} c_{5} \neq 0 \\
c_{3}=0, c_{3}^{\prime} \neq 0\end{array}$ & $\begin{array}{c}c_{3} c_{4} \neq 0, \\
c_{5}=0, c_{5}^{\prime} \neq 0 \\
c_{4} c_{6}-2 c_{5}^{\prime} \neq 0\end{array}$ \\
\hline
\end{tabular}

\section{AdS-horocyclic surfaces associated Frenet frame}

In this section, we will give a example of AdS-horocyclic surfaces to explain that our situation is very different from the ruled surfaces in Euclidean space.

We firstly consider timelike curve as the vanishing AdS-null principle curvature. Let $\gamma$ : $I \longrightarrow H_{1}^{3}$ be unit speed timelike curve with $k_{g}(t) \neq 0$, where $t$ is the arc length parametrization. We consider the Frenet frame $\{\boldsymbol{\gamma}(t), \boldsymbol{t}(t), \boldsymbol{n}(t), \boldsymbol{e}(t)\}$. We define TAdS-horocyclic surfaces associated to the Frenet frame as follows:

Example 7.1 Let $\theta: I \rightarrow[0,2 \pi)$ be a smooth function. We consider two vector fields $\boldsymbol{n}_{\theta}(t)=\cos \theta(t) \boldsymbol{n}(t)+\sin \theta(t) \boldsymbol{e}(t)$ and $\boldsymbol{e}_{\theta}(t)=-\sin \theta(t) \boldsymbol{n}(t)+\cos \theta(t) \boldsymbol{e}(t)$. It follows that $\boldsymbol{n}(t)=\cos \theta(t) \boldsymbol{n}_{\theta}(t)-\sin \theta(t) \boldsymbol{e}_{\theta}(t)$ and $\boldsymbol{e}(t)=\sin \theta(t) \boldsymbol{n}_{\theta}(t)+\cos \theta(t) \boldsymbol{e}_{\theta}(t)$. Therefore, we have $\boldsymbol{n}_{\theta}^{\prime}(t)=\cos \theta(t) \kappa_{g}(t) \boldsymbol{t}(t)+\left(\theta^{\prime}(t)-\tau_{g}(t)\right) \boldsymbol{e}_{\theta}(t)$ and $\boldsymbol{e}_{\theta}^{\prime}(t)=-\sin \theta(t) \kappa_{g}(t) \boldsymbol{t}(t)-\left(\theta^{\prime}(t)-\tau_{g}(t)\right) \boldsymbol{n}_{\theta}(t)$. We consider the pseudo-orthonormal frame $A_{\theta}=\left(\boldsymbol{\gamma}(t), \boldsymbol{t}(t), \boldsymbol{n}_{\theta}(t), \boldsymbol{e}_{\theta}(t)\right)^{t} \in S O_{o}(2,2)$ and define an TAdS-horocyclic surface as follows.

$$
F_{\left(\gamma, t, n_{\theta}\right)}^{T}(s, t)=\gamma(t)+s \boldsymbol{t}(t)-\frac{s^{2}}{2}\left(\gamma(t)+\boldsymbol{n}_{\theta}(t)\right),
$$


By the Frenet-Serret type formula, we have

$$
A^{\prime}=\left(\begin{array}{cccc}
0 & 1 & 0 & 0 \\
-1 & 0 & \kappa_{g}(t) \cos \theta(t) & -\kappa_{g}(t) \sin \theta(t) \\
0 & \kappa_{g}(t) \cos \theta(t) & 0 & \theta^{\prime}(t)-\tau_{g}(t) \\
0 & -\kappa_{g}(t) \sin \theta(t) & -\left(\theta^{\prime}(t)-\tau_{g}(t)\right) & 0
\end{array}\right) A
$$

Then $c_{1}(t)=1, c_{2}(t)=c_{3}(t)=0, c_{4}(t)=\kappa_{g} \cos \theta(t), c_{5}(t)=-\kappa_{g}(t) \sin \theta(t)$ and $c_{6}(t)=\theta^{\prime}(t)-$ $\tau_{g}(t)$. Thus, $F_{\left(\gamma, t, n_{\theta}\right)}^{T}$ is a flat TAdS-horocyclic surface if and only if $c_{4}(t)=-c_{1}(t)$. We assume that $\kappa_{g}(t) \neq 0$, so that the last condition is equivalent to $\cos \theta(t)=-1 / \kappa_{g}(t)$. By Corolary 5.2, $F_{\left(\gamma, t, n_{\theta}\right)}^{T}$ is a front if and only if $\theta^{\prime}(t) \neq \tau_{g}(t)$. This condition is equivalent to

$$
\tau_{g} \neq \pm \kappa_{g}^{\prime} /\left(\kappa_{g} \sqrt{\kappa_{g}^{2}-1}\right)
$$

$F_{\left(\gamma, t, n_{\theta}\right)}^{T}$ is a cuspidal edge at $(0, t)$ if and only if

$$
\kappa_{g} \neq 1, \tau_{g} \neq \pm \kappa_{g}^{\prime} /\left(\kappa_{g} \sqrt{\kappa_{g}^{2}-1}\right) .
$$

$F_{\left(\gamma, t, n_{\theta}\right)}^{T}$ is a cuspidal edge at $\left(s=2 c_{5} / c_{6}, t\right)$ if and only if

$$
\kappa_{g} \neq 1, \tau_{g} \neq \pm \kappa_{g}^{\prime} /\left(\kappa_{g} \sqrt{\kappa_{g}^{2}-1}\right)
$$

$\kappa_{g}^{2}\left(\kappa_{g}^{2}-1\right)\left(\tau_{g}^{2}-2 \tau_{g} \mp 2 \tau_{g}^{\prime} \sqrt{\kappa_{g}^{2}-1}\right) \pm 2 \kappa_{g} \kappa_{g}^{\prime} \sqrt{\kappa_{g}^{2}-1}\left(\kappa_{g}^{2}-\tau_{g}-2\right)+3 \kappa_{g}^{\prime 2}\left(2 \kappa_{g}^{2}-1\right)-2 \kappa_{g} \kappa_{g}^{\prime \prime}\left(\kappa_{g}^{2}-1\right) \neq 0$.

$F_{\left(\gamma, t, n_{\theta}\right)}^{T}$ is a swallowtail at $\left(s=2 c_{5} / c_{6}, t\right)$ if and only if (for simply; we take $\theta^{\prime}=\frac{-\kappa_{g}^{\prime}(t)}{\kappa_{g}(t) \sqrt{\kappa_{g}^{2}(t)-1}}$ )

$$
\kappa_{g} \neq 1, \tau_{g} \neq-\kappa_{g}^{\prime} /\left(\kappa_{g} \sqrt{\kappa_{g}^{2}-1}\right)
$$

$\kappa_{g}^{2}\left(\kappa_{g}^{2}-1\right)\left(\tau_{g}^{2}-2 \tau_{g} \mp 2 \tau_{g}^{\prime} \sqrt{\kappa_{g}^{2}-1}\right) \pm 2 \kappa_{g} \kappa_{g}^{\prime} \sqrt{\kappa_{g}^{2}-1}\left(\kappa_{g}^{2}-\tau_{g}-2\right)+3 \kappa_{g}^{\prime 2}\left(2 \kappa_{g}^{2}-1\right)-2 \kappa_{g} \kappa_{g}^{\prime \prime}\left(\kappa_{g}^{2}-1\right)=0$, $2 \kappa_{g} \kappa_{g}^{\prime} \kappa_{g}^{\prime \prime}\left(5 \kappa_{g}^{4}-2 \kappa_{g}^{3}-7 \kappa_{g}^{2}+2 \kappa_{g}+2\right)+\kappa_{g}^{3} \tau_{g}^{2}\left(\kappa_{g}^{2}-1\right)\left[\left(\tau_{g}-1\right) \sqrt{\kappa_{g}^{2}-1}+1\right]+\left(1-2 \sqrt{\kappa_{g}^{2}-1}\right) \kappa_{g} \kappa_{g}^{\prime 2}+$ $2\left(-6 \kappa_{g}^{4}+4 \kappa_{g}^{3}+5 \kappa_{g}^{2}-2 \kappa_{g}-2\right) \kappa_{g}^{\prime 3}+\left(8 \kappa_{g}^{4}+2 \kappa_{g}^{4} \tau_{g} \sqrt{\kappa_{g}^{2}-1}+2 \kappa_{g}^{3}-2 \kappa_{g}^{2}-4 \kappa_{g} \sqrt{\kappa_{g}^{2}-1}-\kappa_{g}\right) \kappa_{g}^{\prime} \tau_{g} \sqrt{\kappa_{g}^{2}-1}+$ $2 \kappa_{g}^{2} \kappa_{g}^{\prime \prime} \tau_{g}\left(-\kappa_{g}^{4}-2 \kappa_{g}^{3}+2 \kappa_{g}^{2}+2 \kappa_{g}-1\right)-2 \kappa_{g}^{2} \kappa_{g}^{\prime \prime \prime}\left(\kappa_{g}^{4}-2 \kappa_{g}^{2}+1\right)-4 \kappa_{g}^{2} \kappa_{g}^{\prime} \tau_{g}^{\prime}\left(\kappa_{g}^{2}-1\right)-4 \kappa_{g}^{3} \tau_{g} \tau_{g}^{\prime}\left(\kappa_{g}^{2}-\right.$ $1)^{3 / 2}+2 \kappa_{g}^{3} \tau_{g}^{\prime \prime \prime}\left(\kappa_{g}^{2}-1\right)^{5 / 2} \neq 0$.

$F_{\left(\gamma, t, n_{\theta}\right)}^{T}$ is a cuspidal cross cap at $(0, t)$ if and only if

$$
\begin{gathered}
\kappa_{g} \neq 1, \tau_{g}= \pm \kappa_{g}^{\prime} /\left(\kappa_{g} \sqrt{\kappa_{g}^{2}-1}\right) \\
\tau_{g}^{\prime} \neq \pm\left(\kappa_{g} \kappa_{g}^{\prime \prime}\left(\kappa_{g}^{2}-1\right)-{\kappa_{g}^{\prime}}^{2}\left(2 \kappa_{g}^{2}-1\right)\right) /\left(\kappa_{g}^{2}\left(\kappa_{g}^{2}-1\right) \sqrt{\kappa_{g}^{2}-1}\right) .
\end{gathered}
$$

$F_{\left(\gamma, t, n_{\theta}\right)}^{T}$ never be a swallowtail and cuspidal beaks along $\gamma$.

We now consider spacelike curve as the vanishing AdS-null principle curvature. Let $\gamma: I \longrightarrow$ $H_{1}^{3}$ be unit speed spacelike curve with $k_{g}(t) \neq 0$, where $t$ is the arc length parametrization. We consider the Frenet frame $\{\boldsymbol{\gamma}(t), \boldsymbol{t}(t), \boldsymbol{n}(t), \boldsymbol{e}(t)\}$. Without loss of generality, we assume that $\boldsymbol{n}(t)$ is a unit timelike vector. 
Example 7.2 Let $\theta: I \rightarrow \mathbb{R}$ be a smooth function. We consider two vector fields $\boldsymbol{n}_{\theta}(t)=$ $\sinh \theta(t) \boldsymbol{n}(t)+\cosh \theta(t) \boldsymbol{e}(t)$ and $\boldsymbol{e}_{\theta}(t)=\cosh \theta(t) \boldsymbol{n}(t)+\sinh \theta(t) \boldsymbol{e}(t)$. It follows that $\boldsymbol{n}(t)=$ $\sinh \theta(t) \boldsymbol{n}_{\theta}(t)-\cosh \theta(t) \boldsymbol{e}_{\theta}(t)$ and $\boldsymbol{e}(t)=-\cosh \theta(t) \boldsymbol{n}_{\theta}(t)+\sinh \theta(t) \boldsymbol{e}_{\theta}(t)$. Therefore, we have $\boldsymbol{n}_{\theta}^{\prime}(t)=\kappa_{g}(t) \sinh \theta(t) \boldsymbol{t}(t)+\left(\theta^{\prime}(t)-\tau_{g}(t)\right) \boldsymbol{e}_{\theta}(t)$ and $\boldsymbol{e}_{\theta}^{\prime}(t)=\kappa_{g}(t) \cosh \theta(t) \boldsymbol{t}(t)+\left(\theta^{\prime}(t)-\tau_{g}(t)\right) \boldsymbol{n}_{\theta}(t)$. We consider the pseudo-orthonormal frame $A_{\theta}=\left(\gamma(t), \boldsymbol{n}_{\theta}(t), \boldsymbol{e}_{\theta}(t), \boldsymbol{t}(t)\right)^{t} \in S O_{o}(2,2)$ and define an SAdS-horocyclic surface as follows.

$$
F_{\left(\gamma, e_{\theta}, t\right)}^{S}(s, t)=\gamma(t)+s \boldsymbol{t}(t)+\frac{s^{2}}{2}\left(\gamma(t)+\boldsymbol{e}_{\theta}(t)\right)
$$

By the Frenet-Serret type formula, we have

$$
A^{\prime}=\left(\begin{array}{cccc}
0 & 0 & 0 & 1 \\
0 & 0 & \theta^{\prime}(t)-\tau_{g}(t) & \kappa_{g}(t) \sinh \theta(t) \\
0 & \theta^{\prime}(t)-\tau_{g}(t) & 0 & \kappa_{g}(t) \cosh \theta(t) \\
1 & \kappa_{g}(t) \sinh \theta(t) & -\kappa_{g}(t) \cosh \theta(t) & 0
\end{array}\right) A
$$

Then $c_{1}(t)=c_{2}(t)=0, c_{3}(t)=1, c_{4}(t)=\theta^{\prime}(t)-\tau_{g}(t), c_{5}(t)=\kappa_{g}(t) \sinh \theta(t)$ and $c_{6}(t)=$ $\kappa_{g} \cosh \theta(t)$. Thus, $F_{\left(\gamma, e_{\theta}, t\right)}^{S}$ is a flat SAdS-horocyclic surface if and only if $c_{6}(t)=-c_{3}(t)$. We assume that $\kappa_{g}(t) \neq 0$, so that the last condition is equivalent to $\cosh \theta(t)=-1 / \kappa_{g}(t)$. By Corolary 5.5, $F_{\left(\gamma, e_{\theta}, t\right)}^{S}$ is a front if and only if $\theta^{\prime}(t) \neq \tau_{g}(t)$. This condition is equivalent to

$$
\tau_{g} \neq \kappa_{g}^{\prime} /\left(\kappa_{g} \sqrt{\kappa_{g}^{2}+1}\right) .
$$

$F_{\left(\gamma, e_{\theta}, t\right)}^{S}$ is a cuspidal edge at $(0, t)$ if and only if

$$
\tau_{g} \neq \kappa_{g}^{\prime} /\left(\kappa_{g} \sqrt{\kappa_{g}^{2}+1}\right) .
$$

$F_{\left(\gamma, e_{\theta}, t\right)}^{S}$ is a cuspidal edge at $\left(s=-2 c_{5} / c_{4}, t\right)$ if and only if

$$
\tau_{g} \neq \kappa_{g}^{\prime} /\left(\kappa_{g} \sqrt{\kappa_{g}^{2}+1}\right)
$$

$2 \kappa_{g}^{2} \tau_{g}^{\prime}\left(\kappa_{g}^{2}+1\right)^{3 / 2}-2 \kappa_{g} \kappa_{g}^{\prime \prime}\left(\kappa_{g}^{2}+1\right)-2 \kappa_{g}^{3} \kappa_{g}^{\prime} \tau_{g} \sqrt{\kappa_{g}^{2}+1}+2 \kappa_{g}^{\prime 2}\left(3 \kappa_{g}^{2}+1\right)-\left(\kappa_{g} \tau_{g} \sqrt{\kappa_{g}^{2}+1}-\kappa_{g}^{\prime}\right)^{2} \neq 0$. $F_{\left(\gamma, e_{\theta}, t\right)}^{S}$ is a swallowtail at $\left(s=-2 c_{5} / c_{4}, t\right)$ if and only if

$$
\tau_{g} \neq \kappa_{g}^{\prime} /\left(\kappa_{g} \sqrt{\kappa_{g}^{2}+1}\right)
$$

$2 \kappa_{g}^{2} \tau_{g}^{\prime}\left(\kappa_{g}^{2}+1\right)^{3 / 2}-2 \kappa_{g} \kappa_{g}^{\prime \prime}\left(\kappa_{g}^{2}+1\right)-2 \kappa_{g}^{3} \kappa_{g}^{\prime} \tau_{g} \sqrt{\kappa_{g}^{2}+1}+2 \kappa_{g}^{\prime 2}\left(3 \kappa_{g}^{2}+1\right)-\left(\kappa_{g} \tau_{g} \sqrt{\kappa_{g}^{2}+1}-\kappa_{g}^{\prime}\right)^{2}=0$, $\left(\kappa_{g}^{\prime}-\kappa_{g} \tau_{g} \sqrt{\kappa_{g}^{2}+1}\right)^{3}-2\left(\kappa_{g}^{\prime}-\kappa_{g} \tau_{g} \sqrt{\kappa_{g}^{2}+1}\right)\left[2 \kappa_{g} \kappa_{g}^{\prime} \kappa_{g}^{\prime \prime}\left(\kappa_{g}^{2}+1\right)-\kappa_{g}^{\prime 2}\left(\kappa_{g}^{2}+1\right)-\kappa_{g}^{2} \kappa_{g}^{\prime 3}\right]+\left(2 \kappa_{g}^{2}+\right.$ 1) $\left[3 \kappa_{g}^{4} \kappa_{g}^{\prime 3}\left(\kappa_{g}^{2}+1\right)^{-1 / 2}+\kappa_{g}^{2} \kappa_{g}^{\prime \prime \prime}\left(\kappa_{g}^{2}+1\right)+3 \kappa_{g}^{2} \kappa_{g}^{\prime 2}+\kappa_{g} \kappa_{g}^{\prime \prime}\left(2 \kappa_{g}^{2}-1\right)+2 \kappa_{g}^{\prime 2}-\left(7 \kappa_{g}^{2}-1\right) \kappa_{g} \kappa_{g}^{\prime} \kappa_{g}^{\prime \prime}-\kappa_{g}^{2} \tau_{g}^{\prime \prime}\left(\kappa_{g}^{2}+\right.\right.$ $\left.1)^{3 / 2}\right] \neq 0$.

$F_{\left(\gamma, e_{\theta}, t\right)}^{S}$ is a cuspidal cross cap at $(0, t)$ if and only if

$$
\begin{aligned}
& \tau_{g}=\kappa_{g}^{\prime} /\left(\kappa_{g} \sqrt{\kappa_{g}^{2}+1}\right) \\
& \tau_{g}^{\prime} \neq\left(\kappa_{g}^{\prime 2}+\left(\kappa_{g}^{2}+1\right)\left(\kappa_{g} \kappa_{g}^{\prime \prime}-2{\kappa_{g}^{\prime}}^{2}\right)\right) /\left(\kappa_{g}^{2}\left(\kappa_{g}^{2}+1\right) \sqrt{\kappa_{g}^{2}+1}\right) .
\end{aligned}
$$

$F_{\left(\gamma, e_{\theta}, t\right)}^{S}$ never be a swallowtail and cuspidal beaks along $\gamma$. 


\section{References}

[1] V. I. Arnol'd, S. M. Gusein-Zade and A. N. Varchenko, Singularities of Differentiable Maps vol. I, Birkhäuser (1986).

[2] L. Chen, On spacelike surfaces in Anti de Sitter 3-space from the contact viewpoint, Hokkaido Math. J., 38 (2009), 701-720.

[3] L. Chen and S. Izumiya, A mandala of Legendrian dualities for pseudo-spheres in semiEuclidean space, Proc. Japan Acad., 85, Ser. A (2009), 49-54.

[4] L. Chen and S. Izumiya, Singularities of Anti de Sitter torus Gauss maps, Bull. Braz. Math. Soc., 41 (2010), no. 1, 37-61.

[5] L. Chen, Qixing Han, Donghe Pei and Weizhi Sun, The singularities of null surfaces in Anti de Sitter 3-space, J. Math. Anal. Appl.,366(2010) no. 1, 256-265.

[6] S. Fujimori, K. Saji, M. Umehara and K. Yamada, Cuspidal crosscaps and singularities of maximal surfaces. Math. Z., 259, no.4(2008),827-848.

[7] S. Izumiya, D-H. Pei and T. Sano, The lightcone Gauss map and the lightcone developable of a spacelike curve in Minkowski 3-space, Glasgow. Math. J. 42 (2000), 75-89.

[8] S. Izumiya and N. Takeuchi, Singularities of ruled surfaces in $\mathbb{R}^{3}$, Mathematical proceeding of the Cambridge Philosophical Society 130 (2001), 1-11.

[9] S. Izumiya and N. Takeuchi, Geometry of ruled surfaces, Applicaple math. in the golden age, (2003), 305-338.

[10] S. Izumiya, D-H. Pei and T. Sano, Singularities of hyperbolic Gauss maps, Proc. London. Math. Soc. (3) 86 (2003), 485-512.

[11] Izumiya S., Pei D-H. and Romero Fuster M. C., Umbilicity of spacelike surfaces in Minkowski space, Proc. Royal Society of Edinburgh, 134A(2004), 375-387.

[12] Izumiya S., Pei D-H. and Romero Fuster M. C., Singularities of Lightcone Gauss maps of space like surfaces in Minkowski 4-space, Asian Journal of Mathematics, 8, No.3 (2004), $511-530$.

[13] Izumiya S., Kossowski M., Pei D-H. and Romero Fuster M. C., Singularities of lightlike hypersurfaces in Minkowski four-space, Tohoku Mathematical Journal, 58(2006), 71-88.

[14] S. Izumiya, M. Kikuchi and M. Takahashi, Global properties of spacelike curves in Minkowski 3-space, Journal of Knot theory and its Ramifications. 15 (2006), 869-881.

[15] S. Izumiya and M. Takahashi, Spacelike parallels and evolutes in Minkowski pseudospheres, J. Geometry and Physics. 57 (2007), 1569-1600.

[16] Izumiya S. and Romero Fuster M. C., The Lightlike flat geometry on spacelike submanifolds of codimension two in Minkowski space, Selecta Math.(N.S.), 13(2007), 23-55.

[17] S. Izumiya and T. Fusho, Lightlike surfaces of spacelike curves in de Sitter 3-space, Journal of Geometry. 88 (2008), 19-29. 
[18] S. Izumiya, Timelike hypersurfaces in de Sitter space and Legendrian singularities, Journal of Mathematical Sciences. 144, No.1, (2007), 3789-3803.

[19] S. Izumiya, K. Saji and M. Takahashi, Horospherical flat surfaces in Hyperbolic 3-space, J. Math. Soc. Japan 63, No.3(2010), 789-849.

[20] S. Izumiya, T. Nagai and K. Saji, Great circular surface in the three-sphere, Differential Geom. Appl.(2011), doi:10.1016/j.difgeo.2011.02.005.

[21] M. Kokubu, W. Rossman, K. Saji, M. Umehara and K. Yamada, Singularities of flat fronts in hyperbolic 3-space, Pacific J. Math., 221, No.2, (2005), 303-351.

[22] S. Lee, Timelike surfaces of constant mean curvature \pm 1 in anti-de Sitter 3 -space $\mathbb{H}_{1}^{3}(-1)$, Ann. Global Anal. Geom. 29 (2006), 361-407.

[23] M. Maldacena, The Large N Limit of Superconformal Field Theories and Supergravity, Adv. Theor. Math. Phys., 2 (1998), 231-252.

[24] J. Martinet, Singularities of Smooth Functions and Maps, London Math. Soc. Lecture Note Series. Cambridge Univ. Press. 58 (1982).

[25] B. O’Neil, Semi-Riemannian Geometry, Academic Press, New York, (1983).

[26] O. P. Shcherbak, Projectively dual space curves and Legendre singularities, Sel. Math.Sov., 5, no.4, (1986), 391-421.

[27] L. Randall and R. Sundrum, An alternative to Compactification, Physical Review Letters, 83 (1999), 4690-4693.

[28] E. Witten, Anti de Sitter space and holography, Adv. Theor. Math. Phys., 2 (1998), 253291.

[29] H. Whitney, The general type of singularity of a set of $2 n-1$ smooth functions of $n$ variables, Duke Math. J., 10 (1943), 161-172. 